\title{
Über die Sperre der Schlagadern, namentlich der Carotiden, und ihre Folgen.
}

\author{
Von Prof. Wieting, Cuxhaven-Sahlenburg.
}

Bei den Gefahren oder dem Nutzen, den ein künstlich herbeigeführter Gefäßverschluß im Gefolge haben kann, sind die Un ter bindungsitelle als solche und das Endorgandes betroffenen Gefäßes die beiden Hauptfaktoren.

Der erstere Faktor, die Veränderungen an der Unterbindungsstelle, - es soll hier in der Hauptsache nur von Schlagadern die Rede sein - wird sich bei allen Arterien ziemlich gleich bleiben; sekundäre, teils zufällige, teils anatomisch bedingte Besonderheiten wie die mehr oder weniger entfernte nächste Gabelung, die örtlichen Stromverhältnisse, die Nähe infektiöser Herde mit ihren Rückwirkungen und äbnliches können einen gewissen Einfluß auf die Vorgänge an der Unterbindungsstelle gewinnen. Der zweite Faktor, das Endorgan, wird in ganz verschiedener Weise und in ganz verschiedenem Grade betroffen werden je nach seiner histologischen und funktionellen Wertigkeit, seiner Abhängigkeit von der gerade betroffenen Arterie, der Möglichkeit der Kollateralbildung, der funktionellen Tüchtigkeit der vorhandenen Gefäße der Blutzusammensetzung u. a. m. Aus der Vielheit der Komponenten dieses letzteren Faktors allein erhellt, daß die Sperrung der verschiedenen Gefäße auf das Endorgan, auch ohne daß die betreffende Arterie eine sog. Endarterie zu sein braucht, von ganz verschiedener Wirkung sein muß. Wir werden daher in der Erklärung bestimmter Erscheinungen nach Gefä $B$ verschluB uns von vornherein $z$ u hüten haben, alle solche Folgeerscheinungen einheitlich erklärenzu wollen.

Dic noch immer umstrittenen Folgeerscheinungen der S per rung einer Carotis communis bedürfen bei ihrer vitalen Bedeutung möglichst allseitiger Beleuchtung: Perthes hat kürz- 
lich auf der Chirurgentagung 1920 und dann in einer Arbeit in v. La ngenbecks Archiv f. klin. Chir. 1920, Bd. 114: „Uber die Ursache der Hirnstörungen nach Carotisunterbindung und über Arterienunterbindung ohne Schädigung der Intima" diese Frage wieder angeschnitten : er sieht die Ursache der Hirnstörungen vornehmlich in embolisch-thrombotischen Vorgängen, die zu der Unterbindungsstelle in engste Beziehung gestellt, während andere Momente nur kurz und meist ohne bestimmte Stellungnahme zu ihnen gestreift werden.

Auf dem gleichen Chirurgentag wurde in der Diskussion von Klapp (s. Sitzungsbericht) darauf hingewiesen, daß das, was für die Carotis gelte, doch auch für die anderen Arterien, z. B. die der Extremitäten gelten müsse: Das ist, vom pathologisch. anatomischen Standpunkt aus gesehen, auch durchaus zutreffend, in Übereinstimmung mit dem oben aufgestellten Satz, daß die histologischen Veränderungen an der Unterbindungsstelle überall dieselben sein müssen, wenn sie durch dieselbe Schädigung hervorgerufen werden, und ein Embolus kann sich von jedem sich bildenden Thrombus unter gleichen Stromverhältnissen los. reißen und fortgeschwemmt werden. Nur sind die Wirkungen a uf das Endorgan sehr verschieden: sie treten beim Gehirn weit rascher und schwerer in Erscheinung, als z. B. an den Extremitäten, und werden darum auch z. B. nach Femoralisunterbindung nicht im Sinne Perthes sich auswerten lassen. Da also die Veränderungen an der Unterbindungsstelle nicht ohne weiteres und einheitlich fürdie Folgen einer Unterbindung verantwortlich ge. macht werden können, müssen diese wenigstens zum Teil sich aus der Eigenartdes Endorgans herleiten lassen: dieses spielt also zweifellos eine wesentliche Rolle für die Folgen auch rein thrombosierender oder embolischer Vorgänge.

Die erste Fragestellung nun wäre die: Kommt tatsächlich die Embolie nach Carotisunterbindung so häufig vor und woraus ließe sich diese herleiten? Und dann, ist sie von solcher Wichtigkeit und solcher Natur, daß die Art der Gefäßunterbindung dem Rechnung tragen müsse?

Das VorhandenseineinesThrombusist zunächst 
die Vorbedingung für sein Weiterwachsen wiefür e ine Embolie a us ihm. Wir kämen hiermit auf das so außerordentlich verwickelte und interessante Thema der Thrombenbildung überhaupt, der Stromverhältnisse der Gefäß. wand- und Gefäßinhaitsveränderung in ihren wechselseitigen Beziehungen. Aus diesem Gebiet, das vortrefflich in $\mathrm{Mar} \mathrm{ch}$ a nd. $\mathrm{Krehls}$ Handbuch der allgemeinen Pathologie, Bd. II, dargestellt ist, nehme ich hier das Wichtigste heraus, was unsere Fragestellung beantworten helfen könnte.

Die histologischen Verhältnisse, die sich nach Gefäßunterbindung an der Unterbindungsstelle abspielen, dürften, wie ich vielfach erfahren habe, gerade chirurgischerseits oft nicht genügend bekannt und gewürdigt sein: immer wieder trifft man auf die Ansicht, daß zur endgültigen Gefäßverschließung ein Thrombus benötigt werde, der meistens bis zur nächsten Gabelung gehe. Marchand sagt darüber in seinem Werke „Der ProzeB der Wundheilung" Igor, etwa Folgendes in histologis cher Beziehung:

$\mathrm{Nach}$ der Unterbindung einer Arterie kommt die Heilung in der Regelohne Bildung eines gröBeren Thrombus zustande, indem die einander eng anliegenden Gefäßwandungen verkleben; das Endothel überzieht in sehr kurzer Zeit die sehr geringe durch etwas Fibrin ausgefüllte Spalte. In anderen Fällen, bei stärkerer Rauhigkeit des Arterienrohres, Arteriensklerose, mangelhafter Zirkulation bildet sich ein größerer Thrombus an der Unterbindungsstelle. Ganz ähnlich verhält sich die Unterbindung in der Kontinuität: auch hier kann eine Thrombenbildung so gut wie ganz ausbleiben, während sich in anderen Fällen ein zentraler und, bei ungünstigen Zirkulationsverhältnissen, mangelhaftem Kollateralkreislauf, besonders bei älteren Individuen mit Arteriosklerose auch ein ausgedehnter peripherer Thrombus bilden kann. In Übereinstimmung damit steht die Heilung glatter Gefä $B$ wunden ohne Unterbindung. Die Gefäßwand, abgesehen von der Adventitia, entbehrt der eigenen Gefäße. Das Fibrin, das die Verklebung herbeiführt, wird von dem im Gefäßlumen strömenden Blute geliefert, nachdem sich an der verletzten Stelle ein Blutplättchenthrombus gebildet hat. Mit dem Fibrin werden farblose und rote Blutkörper- 
chen abgelagert, so da $B$ sich ein größerer Thrombus bilden kann, der das Lumen teilweise oder ganz abschließt. Indes ist die Bildung eines größeren Thrombus für die Heilung einer Gefäßwunde keineswegs erforderlich, vielmehr ist der normale Vorgang der, daß die Wunde sich schließt, ohne daß das Gefäßlumen erheblich beeinträchtigt wird.

Diese im wesentlichen experimentell bei aseptischem Wundverlauf gefundenen Tatsachen werden von $\mathrm{p}$ athologis $\mathrm{ch}$. an atomischer Seite bei Verletzungen, und hier namentlich bei Schußverletzungen, durch A. Dietrich stark gestützt und ihm verdanken wir auch eine gut vorstellbare Begriffsfestlegung, von der $z$ u erwartenden GröBederetwaigen Throm. ben. Dietrich fand unter anderem in 6I Proz. seiner 115 frischen und älteren Amputationsstümpfe, die teils aus Leichen, teils von amputierten Gliedern gewonnen wurden, in den unterbundenen Arterien entweder gar keinen ( $=0 \mathrm{~mm}$ in 36 Fällen) oder nur einen geringfügigen (unter $3 \mathrm{~mm}$ in 34 Fällen) Verschluß. pfropf; bei Frühamputationen überwiegt das Ausbleiben eines Thrombus. Ein Thrombus von $\mathrm{I} \mathrm{cm}$ und darüber fand sich nur bei örtlich eitriger Infektion am Amputationsstumpf. Schon daraus erhellt die Bedeutung derInfektion fürdieThrombenbildung, auf die auch ich in früheren Abhandlungen über Wundinfektionen (s. Deutsche Zeitschr. f. Chir., Bd. I 46 u. 150) hinwies. Ich konnte vielfach Dietrichs Befunde bestätigen, $\mathrm{da}$ wir uns gemeinsamer Arbeit in gemeinsamer Interessengemein. schaft auf längere Zeit während des Krieges erfreuen durften.

Auf die biologischen Vorgänge bei der Thrombenbildung geht Beneke in Ma rchand-Krehls Handbuch gründlichst ein. Er unterscheidet den Stagnations- und den Pulsions. thrombus. In beiden Fällen kommt es im wesentlichen auf die Beeinflussung des Gefäßinhalts an, die ihrerseits von dem Zustand der Gefäßwand ausgeht. „Thromben entstehen erst, wie bei der Stase durch besondere Einwirkungen der noch lebenden, wenn auch absterbenden Gewebe auf das ruhende Blut, sowie durch Zuwanderung und Ansammlung von Leukocyten, die dann im Lumen absterben. Die Entstehung der roten Stagnationsthromben (z.B. nach Venenunterbindung) wird durch zwei Momente bestimmt: die Abschwächung bzw. den Mangel der 
Blutbewegung und das lokal verfügbare Quantum der dieBlutnekrose bzw. Fibringerinnung bewirkenden oder hemmendenSubstanzen. Die weiBen Pulsionsthromben nach Arterienunterbindung entstehen nach mechanischen Gesetzen auf Grundlage der Stromverlangsamung, Wirbel- und Wellenbewegung. Man gewinnt aber den Eindruck, als ob chemotaktische, von der Agglutination ausgehende Reizungen die Anlagerung der Leukocyten und ihren auffallenden jähen Zerfall unterstützten. Die Fibrinanlagerung erfolgt anscheinend unter chemischer Beteiligung der zerfallenden Leukocyten, von dem kongelatinierten Zentrum aus, je nach den Bedingungen der Blutströmung; bei sehr flot te m Strom bleibt sie a us, bei schwächerem tritt sie ein." Aber so stark auch das ursächliche mechanische Moment in den Formen der Pulsionsthromben zum Ausdruck kommt, man darf darübernicht vergessen, da Bder Zelluntergang mitseinenchemischen Ursachen und Folgen von Anfang an ein integrierendes Glied inderGeschichteauchdieser Pfropf. bildung darstellt.

$\mathrm{Daß}$ nun in Experiment und in der chirurgischen Praxis an der Stelle der bleibenden Unterbindung so geringfügige, für Embolie schon aus diesem Grunde wenig geeignete Thrombenbildung platzgreift, trotz mechanischer Stromänderungen, mag m. E. mit daher rühren, daß das Blut, der Gefäßinhalt, mit der durch Schnürung etwa geschaffenen (aseptischen!) Gefäßwandnekrose nur in sehr geringem Umfang in Berührung kommt und demnach die chemisch biologische Einwirkung der zugrunde gehenden Gewebselemente mit dem Gefäßinhalt recht unbedeutend ist. Umschnürt man eine Arterie mit einem runden Faden, so formt sich das Gefäßrohr unter Eigenkontraktion diesseits wie jenseits zu einem gefältelten Trichter, der sich nach der Unterbindungsstelle $z u$ verjüngt: Intima wird überall die Intima berühren, sofern der Blutstrom wirklich unterbrochen ist. Dabei braucht oder soll vielmehr eine schwerere Schädigung der Gefäßwand gar nicht zustande kommen, denn es ist ja eine alte chirurgische Regel, wenigstens bei allen Unterbindungen in der Kontinuiät, den Faden nursofestzuschnüren, daB ebender Blutstrom unterbrochen bleibt. Beider 
Unterbindung durchtrennter Gefäßenden wird sich eine schwerere Läsion der Intima freilich kaum vermeiden lassen, will man gegen eine Nachblutung genügend gesichert sein. Immerhin wird ge nügende Vorsicht auch hier die Intima nur so wenig schädigen, $\mathrm{da}$ zur ausgedehnteren Thrombenbildung keine Veranlassung geschaffen wird. Auch die Vorgänge bei breiter Aufeinanderpressung der Gefäßwände etwa durch Klemmen, Gummischnürung über nachgiebigere Fremdkörper, Tamponade zum Venenverschluß u. a. m. näher einzugehen, erübrigt sich hier.

Diese Betrachtung diene als Unterlage unserer Erkenntnis, da $B$ die gewöhnliche GefäBunterbindung, als bleibende gedacht, a priori nicht die Gefahren in sich schlieBt, die aus Thrombose und Embolie entstehen könnten. Das gilt auch fürdie einfache: Carotisunterbindung; es werden nennenswerte Thromben sich a uch bei ihr nicht bilden und darum a uch keine Emboli - unternormalen Umständen. Wenn sie sich aberdennoch bilden sollten, so müssen auBergewöhnliche Umstände vorge. legen haben.

In der großen Mehrzahldieser Fälle dürfte es sich in erster Linie um eine komplizierende Infektion $\mathrm{h}$ andeln. Es kommt hier weniger die agglutinierende Wirkung der im Kreislauf bei den Infektionskrankheiten sich findenden Toxine in Betracht, als der Entzündungsproze $B$ in der Gefäßwand selber, der schon extravaskulär im Bindegewebe beginnt und sich endothelwärts fortsetzt mit seiner Bildung sero-fibrinösen Exsudats und Leukocytenansammlung bzw. Leukocytolyse, die den wesentlichen Ansto $B$ zur Fibringerinnung und Plättchenagglutination auf der erkrankten Stelle abgibt. Mechanische Stromveränderungen begünstigen weiterhin die Thrombenbildung, wie das oben nach Beneke ausgeführt wurde. Wenn ich nun auch nicht der Ansicht beipflichte, daß jede Arterienthrombose nach Unterbindung auf Infektion zurückzuführen ist, so ist sie es praktisch doch in den allermeisten Fällen.

In zweiter Linie kommt allerdings die mehr oder weniger große Mißhandlung der Gefäßwand bei der Unterbindung in Frage, aber auch hier wieder in erster Linie in 
dem Sinne, dalis durch solche Mißhandlung die Bedingungen für eine Infektion eher gegeben sind als bei schonendem Vorgehen, in zweiter Linie das rein mechanische Moment an sich und in seinem Verhalten zum Blutstrom und zum GefäB. inhalt.

Nehmen wir einmal die Unterbindung in der Konti$n$ u it ät: Hat man aus diesem oder jenem Grunde das Gefäß vorher mit einer harten Klemme abgeklemmt, so daß an dieser Stelle eine schwere Schädigung der Gefäßwand, besonders der Intima, entsteht und legt man z. B. an der Canotis nachher einen Unterbindungsfaden an der Stelle so an, daß die stark gequetschte Stelle in den peripheren Trichter der Unterbindungsstelle fällt, dann sind allerdings die Bedingungen für eine Thrombenbildung weit eher gegeben, als bei der einfach unterbundenen Arterie, um so mehr als auch die Infektionsbedingungen durch das gleiche Trauma erhöht wurden. Bei durchschnittenen Arterien fällt diese Gefahr ja meist von selbst fort, da man diesseits der Klemme unterbindet. Man soll also schon aus diesem Grunde bei der GefäBunterbindung möglichst jede Ge$f a ̈ B m i B h$ andlung vermeiden, soll vor allem auch die Läsion der Adventitia möglichst vermeiden, soll nicht $\mathrm{zu}$ sehr isolieren, denn die Adventitia führt die Vasa vasorum, und ist $f \ddot{u} r$ das Gefä $B r o h r$, was das Periost $f$ ür den $\mathrm{Knoch}$ en ist. Handelt man so, dann werden Thromben an der nichtinfizierten Unterbindungsstelle eine Seltenheit bleiben. Die Struktur der Gefäße spielt gewiß auch eine Rolle; es werden atherosklerotische Gefäße durch Unterbindung stärker geschädigt als junge elastische regenerationsfähige, und somit allein würden das Alter, aber auch die Allgemeininfizierten, die Anämischen (s. u.) mehr gefährdet sein als gesunde kräftige Menschen.

Bei der Bewertung von a $\mathrm{n}$ de $\mathrm{r} \mathrm{L}$ e i $\mathrm{c}$ h e vorgefundenen roten Thromben, die sich an eine Unterbindungsstelle bzw. einen derberen älteren Thrombus im Gefäßlumen anschließen - ich setze voraus, daß Thromben und nicht etwa postmortale Gerinnsel vorlagen, - müßte doch immer auch die Frage aufgeworfen werden, die ich zwar nirgendwo aufgeworfen finde, ob solche Thromben im Einzelfalle auch wirklich ursächlich für die vorhanden gewesenen Hirnstörungen bzw. den Tod verantwortlich gemacht 
werden dürfen. Es liegt doch manchmal der Gedanke nahe, daB manche solcher Schwerverletzten oder Schwererkrankten nicht sterben, weil sie Thromben haben, sonderndaB sie Thromben haben, weil sie sterben. Ich führte den ähnlichen Gedankengang bereits früher in meinen Arbeiten über die Wundinfektionen (l. c.) aus, da $B$ auch manche Menschen nicht sterben, weil sie septisch werden, sondern septisch werden, weil sie sterben, wie H. Frits ch (Bonn) uns als seinen Schülern einprägte. Für solcher Art Thrombenbildung wäre eine Infektion natürlich nicht immer erforderlich, aber auch die Art der Unterbindung ganz bedeutungslos. Mit Sicherheit läßt sich zwar eine solche Beziehung zwischen Tod und Thrombenbildung bzw. Infektion niemals erweisen, aber solche Umkehrung der in unserer Vorstellung eingewurzelten Abhängigkeit oder auch nur ihr Gleichgeordnetsein ist nicht von der Hand zu weisen.

Angenommen nun, es habe sich ein $\mathrm{Th}$ rombus, der das Gefäßlumen mehr oder weniger ausfüllt, gebildet also ein Throm-

Anmerkung: Es ist natürlich nicht nötig, daß bei der infektiösen Thrombose die Unterbindungssstelle von Eiter umspült sei, es genügt schon eine sog. leichte, mit bloßem Auge vielleicht gar nicht erkennbare Infektion, z. B. vom Faden auf die umschnürte Gefäßwandung übergehend, um die Thrombenbildung einzuleiten und das um so mehr als eine Infektion manchmal recht schnell innerhalb weniger Stunden sich geltend machen kann. Man könnte den ganzen ProzeBder Throm. benbildung, welcher sich im GefäBlumen unter Wechselwirkung zur und von der Gefäßwand abspielt, teleologisch als eine Abwehr des Blutes selber und des Endothels, als eine Entzündung flüssigen Gewebes ansehen, die der entzündlichen Reaktion des festen Gewebes von ihrer leichten fibrinösen Ausscheidung bis zur Eiterbildung gleichzusetzen wäre. Ich habe bei schwerer örtlicher infektiöser Phlebitis selbst Eit er. füllung der Venenlumina a uf weite Strecken hin ohne eigentliche Thrombenbildung beschrieben, die nicht gleichzusetzen ist dem eitrigen Zerfall eines vorher solideren Thrombus, und zwar sowohl an den Venen der Extremitäten wie an der Carotis int., in letzterem Falle mit tödlichem Ausgang (Fall 7).

Die Kriegsbeobachtungen, namentlich frischer Fälle, lassen natürlicherweise die mikroskopische Untersuchung meistens vermissen und sind somit, selbst wenn sie von scharfen Beobachtern stammen, wie z. B. K roh, nicht recht beweiskräftig und einwandsfrei gegenüber genauer untersuchtem Material. 
bus meist auf infektiöser Grundlage, so kann dieser wach. sen, kann das Lumendes unterbundenen Gefäßes ganz ausfüllen und zu Gefäßgabelungen a uf-oder absteigen.

Ich habe eine solche das Leben gefährdende, anscheinend nicht infektiöse Thrombenbildung abgebildet und beschrieben (s. Deutsche Zeitschr. f. Chir. I 907, Bd. 90: „Über den Nutzen und dic Gefahren der der ein- und doppelseitigen Oberkieferresektion vorausgeschickten Carotisunterbindung").

Es hatte sich innerhalb 24 Stunden nach der Operation, nach Unterbindung der Carotis externa nahe der Bifurkation ein Thrombus gebildet, der die Carotis communis und die Carotis interna völlig ausfïllte. Es hatte vorher, $d . h$. vor der eigentlichen Unterbindung der Externa, eine st arke $Q$ uetschung der Communis stattgefunden durch cine starre Klemme, wie das Präparat später zeigte, die Intima und die Muscularis an einer Stelle völlig durchtrennt hatte. Die völlige $W$ egsperrung an der $G$ abelung wardie Ursachefür die tödlicheHirnstörung, dicetwa $20 \mathrm{Std}$. nach der Operation a uftrat, nicht etwa ein Embolus oder in die Schenkelhöhle einwachsender Thrombus, wie dic Autopsic ergab. Entzïndliche Erscheinungen fehlten makroskopisch ganz. Ich habe damals die mechanische GefäBwandverletzung als alleinige Ursache der örtichen Thrombose angesehen und sie mag auch wohl die wesentlichste Rolle gespielt haben für diese im Blutstrom sich bildenden Veränderungen des Gefäßinhalts. Aber ich habe nun dic mir zur Verfügung stehenden Präparate noch cinmalder Durchsicht unterzogen, um zu sehen, ob dem makroskopisch negativen Befund auch ein solcher mikroskopisch entspreche. Die Durchmusterung zeigte nun, daß zwar die Carotis externa an der Unterbindungsstelle frei ist von Infiltration, daß auch die Fäden im Querschnitt nach dem Lumen zu reizlos liegen; aber schon auf ihrer Rückseite, d.h. ihrer freien Peripheric, tragen sie reichliche Leuko-

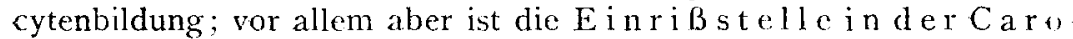
tis communis an der Lücke, wo nur die Adventitia steht, starkmit Lcukocyten infiltriert auf etwa einen Zentimeter zentralwärts hin abklingend, während die gegenüberliegende Wand im Schnitt noch frei ist. Auch der Thrombus zeigt hier an sciner offenbaren Entstehungsstelle stark leukocytäre $Z$ usammensetzung. Die Infiltration an der Läsionsstelle ist so stark, daß an ihrem infektiösen Charakter nicht gezweifelt werden kann; auch auf der A. thyreoidea sup., die am Knoten liegt, ist in der Adventitia die gleich starke Infiltration, hier schon anscheinend ein freies fibrinös eitriges Exsu- 
dat bildend, das man bei genauerem Zusehen wohl auch makroskopisch hätte entdecken müssen.

Ich muB also auch in diesem Falle der infehtiösen KomponenteeinegröBereRollezusprechen, als ich damals tat, wo mir dieser Zusammenhang noch nicht so klar war, und ich muß weiter den Schluß daraus ziehen, daß die Mitwirkung einer Infektion nurdurch mikroskopische Untersuchung, am besten auch bakteriologische, a usgeschlossen werden kann, die makroskopische allein aber nicht dazuberechtigt.

Wenn wir nun eine Carotis communis unterbinden, so bleiben wir doch in der Regel - oder sollten es doch - meh. rere Zentimeter von der Gabelung entfernt und ein Hineinwachsen eines Thrombus in die Gabelung bis zur Verlegung der Interna dürfte, wenigstens ohne Infektion, recht selten sein: Jedenfalls würden Stunden oder Tage dazu gehören, also $\mathrm{klinis} \mathrm{ch}$ e in freies Intervall, wenn wir Perthes' Ansicht, daß bei der Unterbindung der Communis der rückläufige Strom aus der Ex. terna zur Interna zur Ernährung des Gehirns genügen soll, beipilichten wollten - was ich, nebenbei bemerkt, nicht ohne weiteres tue. Perthes muß also, da die Absperrung der Communis für sich ihm nicht genügt zur Ernährungsstörung des Gehirns, auf ein anderes Moment denken und sieht ein solches in der Em. bolie von der Unterbindungsstelle her - es mag aber, nachdem er anatomisch einmal ein solches Vorkommen, bei einem spontanen echten Aneurysma der Carotis interna, in dem natürlich sog. blande Thromben vorhanden waren, nach der Operation beobachtet zu haben glaubt, sein Gedankengang auch umgekehrt gewesen sein.

Ich bezweifle aber das häufige Vorkommen einer Embolie von einem einfachen Unterbindungsthrombus der Carotiden her. DaB ein Abreißen einmal vorkommen $\mathrm{k}$ a $\mathrm{n} \mathbf{n}, \mathrm{z}$. B. durch heftige Halsbewegung, beim Erbrechen, durch brüskes Manipulieren am Halse während oder nach einer Operation, wenn schon ein Pfropf, wie beim spontanen Aneurysma verständlich, vorhanden war (s. u.), ist ohne weiteres zuzugeben. Aber sicherlich sind das Seltenheiten. Es fehlt ja bei dem unterbundenen Gefäß die Vis a tergo, die den Thrombus 
zerreißen und fortschwemmen könnte, und der eventuelle rückläufige Strom aus der Externa zur Interna, so lange diese Bahn offen ist, ist doch wohl zul schwach, um nennenswert in Frage zu kommen.

Leichter denkbar und möglich ist natürlich das Hineinwachsen eines Thrombus, doch ist zu bedenken, daß bei unterbundener Communis erst dann seine, $d . h$. ihm zur Last zu legende deletäre Wirkung auf das Gehirn eintreten könnte, wenn er Schlimmeres anrichtete als die Unterbindung der Communis und das würde nach Perthes' Darstellung der Verhältnisse wohl erst dann eintreten, wenn er bis zur Schädelhöhle gelangt wäre und den Circulus Willisii erreicht hätte. Solches k a n n freilich bei infektiöser Thrombose namentlich auch ziemlich rasch sich vollziehen, aber dann werden die Erscheinungen der schweren Infektion doch deutlich sein, und das klinische Bild der Thromboarteriitis wird sich in seiner Entwicklung über einseitige Amaurose mit sonstigen Augenerscheinungen, wie Schmerzen, Lidschwellung, Pupillendifferenz usw. meistens wohl kundtun, bis es zur schwereren Hirnstörung kommt und diese wird dann durchaus nicht immer als solche zum Tode führen, sondern eher die Allgemeininfektion oder andere Komplikationen.

Ich habe einen solchen Fall (s. Fall 7) mit Granatsplitterverletzung der $\mathrm{A}$. carotis communis und $\mathrm{V}$. jugul. int. nekroptisch verfolgen können, nachdem ich zweimal wegen Blutung und Spätblutung septischer Natur die Communis ohne Folgen für das Gehirn unterbunden hatte; aber nach der dritten Spätblutung bildete sich im Verlauf mehrerer Tage über Chemose und gleichseitiger Erblindung eine Halbseitenlähmung aus, die über a u f steigende eitrige Endoarteriitis (nicht gleichbedeutend mit eitrigem Throm. benzerfall!) bis zur Carotis cerebralis unter septischen Allgemeinerscheinungen und crupöser Pneumonie zum Tode führte.

In zwei weiteren Fällen nach Granatsplitterverletzung der Carotis interna bzw. cerebralis, bei denen die Blutung nur durch feste Tamponade und Hautübernähung zum Stehen gebracht werden konnte. entwickelte sich ebenfalls nach mehreren Spätblutungen im Verlauf weniger Tage eine Halbseitenlähmung, offenbar infolge aufsteigender infektiöser Thromboarteriitis; doch genasen beide $F$ älle nach mehrmonatigem Krankenlager schließlich und konnten fieberfrei und unter fortschreitender Besserung der Symptome in die Heimat entlassen werden. 
Ich komme also zu dem Ergebnis, daB die Anschauung Perthes' betreffend Embolie für die Dauer Unterbindungsfälle an vorher noch nicht geschä digten bzw. nicht thrombushaltigen Arterien als häufiger Vorgang nicht zutrifft, a uch nicht für die arteriosklerotischen Gefäße.

Etwas anders liegen die Dinge nun nach einer temporären Gefäßabsperrung und bei Operationen an schon thrombenhaltigen Arterien. Sie liegen schon deshalb anders, weil diese Gefäße wenigstens einstweilen, bis zu ihrer endgültigen Versorgung, im Blutstrom eingeschaltet liegen bleiben und etwa vorhandene Thromben der Vis a tergo ausgesetzt sind, so daß sowohl Abriß wie Einschwemmung peripherwärts leicht erklärlich sind.

Es kommen hier praktisch zwei Dinge in Betracht. Erstens haben wir bei unseren Operationen an noch gesunden Gefäßgebieten, die aber temporär aus diesem oder jenem Grunde gespert werden müssen, z. B. zwecks Blutsparung oder Sicherung des Operationsverlaufes, die Sperrung so schonend wie möglich zu machen, so daß die Gefäßwand nach Lösung der Sperre intakt bleibt. Es ist das ja eine alte Regel, auf die auch Pels-Leusden in der Diskussion über Perthes' Vortrag hinwies, daß man die temporäre Sperre nur soweit steigert, daß eben gerade das Ziel, den Blutstrom zu unterbrechen, erreicht wird (s. auch Pels-Le usden, Chirurgische Operationslehre, 2. Aufl.). Auf die vielen schonenden Methoden, die angegeben sind, will ich hier nicht weiter eingehen. Ich selbst habe z. B. bei den arterio-venösen Anastomosen der Femoralgefäße der Raumenge wegen über ein durch ein entsprechend geformtes Korkstück gespanntes Gummirohr von Gefäßkaliber mit dickem Catgutfaden locker gesperrt, oder ich benutze zur temporären Sperre einer größeren Arterie eine feine nach Art der Kocherschen Darmklemme konstruierte GefäBklemme, deren Gabel durch Jodoformmullwicklung oder Gummiröhrchen geschütz: sind und deren freic Enden feine Zähnung tragen, um gegen das Abrutschen sich und das gesperrte Gefäß an benachbartes Bindegewebe oder Muskel anzuhaken (Beschreibung 5 . 
Deutsche med. Wochenschr. I go8, H. 28: „Die angiosklerotische (iangrän usw.").

Wird nun aber bei solcher temporären Sperre Intima und Muscularis stärker geschädligt, wie $\mathrm{P}$ e r th es es abbildet, so kann sich wohl auf dieser mechanischen Grundlage, unter Berücksichtigung der chemotaktischen Einflüsse im Sinne Benekes (s. o.) ein wandständiger Pulsionsthrombus bilden. Die gleiche Grundlage wird natürlich geschaffen durch gewalttätige Verletzung durch Stich oder SchuB, wie das ja bekannt ist, sei nun die Intima durch vorbeistreifende Granatsplitter allein oder mitsamt der Muscularis eingerissen, oder sei eine stärkere Gefäßwandschädigung mit Bildung eines kommunizieren. den Hämatoms, dem sog. falschen Aneurysma, entstanden. Bei letzteren bilden sich wohl immer Thromben in dem sog. Aneurysmasack, der ja in Wirklichkeit kein Sack ist ( $\mathrm{s}$. Voll brech t. Wieting, Kriegsärztliche Erfahrungen aus den Balkankriegen, 1914) und es ist wohl verständlich, daß in letzterem Falle die Strömungsveränderungen als Verlangsamung oder Wirbelbildung in Verbindung mit der schweren Wandläsion allein, vor allem aber durch den Kontakt des Blutes mit der zerwühlten Muskelsubstanz (Thrombokinase) zur Thrombenbildung führen kann. Ich möchte aber doch auch hier, für alle anderen Fälle, z. B. der einfachen Intimaquetschung, auf die Bedeutung gleichzeitiger Infektion in der Umgebung der Wand hinzuweisen nicht versäumen, und glaube, daß diese auch in klinisch als nichtinfiziert geltenden Fällen, doch bakteriologisch und histologisch betrachtet, eine Rolle spielen mag, wo man sie bisher ausschloß oder nicht daran dachte. Bei Schußwunden, zumal durch Granatsplitter, wird dieser Zusammenhang angesichts ihrer Natur ja stets begreiflich erscheinen. Prak. tisch aber müssen wir bei unseren aseptischen Eingriffen auch von diesem Gesichtspunkte aus mehr die Infektion als die traumatische Gefäßschädigung fürchten lernen.

Man wird Beneke beipflichten müssen, daß beständige starke Strömung hemmend auf die Thrombenbil. d ung wirkt, trotz sonst gegebener Vorbedingungen, wenn sic sie auch nicht ganz zu verhindern vermag. Dieser Umstand er klärt auch wohl mit das hä ufige A usbleibender Throm. bosierung nach zirkulärer oderseitlicher Gefäß. 
$\mathrm{n}$ a $\mathrm{h} \mathrm{t}$, falls der Verlauf sonst normal ist; selbst der Einfluß leichter Infektion mag durch die Strömungsenergie aufgewogen werden können. Bilden sich dennoch nach einer Gefäßnaht obturierende Thromben, so ist entweder stärkere Infektion oder allzu starke Lumenverengerung (relativ zur Herzkraft, Blutdruck usw.), oder, wie meist, beides im Spiel. Die wirksamen Einflüsse der Infektion auf die Thrombenbildung und Embolie werden von $\mathrm{Be}$. $\mathrm{neke}$ (1.c.) eingehend besprochen und neuerdings hat D i e trich (Cöln) an umfangreichem Material diese Dinge für Kriegswunden vom pathologisch-anatomischen Gesichtspunkt aus gründlichst bearbeitet (s. D i e trich s Monographie I9Io: „Die Thrombose nach Kriegsverletzungen" und Münchner med. Wochenschr. I920, Nr. 32 : „Kriegserfahrungen über Thrombose").

Bildet sich nun aus diesem oder jenem Grunde im strömenden Blute ein Thrombus, so kann die. sernatürlich sowohl durch Umfangszunahmedas Lumen versperren und als kontinuierlicher Thrombus peripher weiterwachsenals a uch durch AbriB zur Embolie führen. Die Emboli werden dann entweder als solche gefährlich, also vom Augenblick der Einschleppung an, oder sie werden gefährlich durch zu beiden Seiten sich anschließende sog. Sekundärthromben, die ihrerseits wieder Gefäßgabelungen sperren können (Beneke, l. c. „Die Embolie"). Hierauf fußend darf ich auf einen klinisch wichtigen Unterschied bezüglich des sog. freien In tervalls hinweisen: Erfolgt nach einer bleibenden Unterbindung einer örtlich gesunden Carotis communis die schwere Hirnstörung sofort, dann kann es sich nicht um eine Embolie handeln, da ja ein Thrombus noch nicht vorhanden war, ein solcher bis zu seiner gefährdenden Entwicklung immerhin einige Stunden beanspruchen würde. Das $\mathrm{Nichtvorhandensein} \mathrm{eines}$ freien Intervalls ist aber andererseits kein $\mathrm{Be}$ weis für die nichtembolische Natur einer Hirnstörung: Manipulieren wir an ein em vorhergeschädig. ten Gefä $B$ rohr, in dem sich schon ein Thrombus befand, nah oder fern der Operationsstelle, so kann dieser durch unsere Manipulation, wie den bloßen Fingerdruck, die Stromsperre und Wiederfreigabe usw. zum Embolus werden, und es tritt dann die 
schwere Hirnstörung ebenfalls unmittelbat im Anschlu B an die Operation ein. Es brauchen das durchaus nicht gerade falsche Aneurysmen sein, wie im Falle Perthes, sondern auch einfache Läsionen der Gefäßwand, namentlich in Verbindung mit Infektion, liefern solche gar nicht seltenen Fälle.

Ich habe einen solchen Fall (8) operiert, in dem nach Unterbindung der Vena jugularis und der Carotis int. bei pulsicrendem Hämatom dieser und vollig durchschossener aber beiderseits thrombotisch verschlossenen Carotis externa, unvermuteterweise sofort nach der Operation schwerste Hirnstörungen: Bewußtlosigkeit mit Halbseitenlähmung eintrat. Dic Autopsie des nach wenigen Stunden Verstorbenen ergab cine $\mathrm{Kmbolic}$ der Cerebralis media mit Sekundärthrombose der kleinen Äste, ausgehend von cinem Pulsionsthrombus, der sich an der lädierten und infizierten Verletzungsstelle der $\mathrm{C}$. int. gebildet hatte.

Über cinen ähnlichen Fall berichtete ich kürzlich, indem ich eine Erweichung des Halsmarks auf einen thrombotischen Vorgang in einer Vertebralarterie zurückführen konnte. (S. Deutsche Zeitschr. f. Chir. 192I, Bd. I64.)

Darin ist Perthes beizupflichten, daß, wenn ein freies Intervall zwischen Unterbindung einer Carotis und der Hirnstörung nachweisbar ist, diese Schädigung nur durch Embolie oder Thrombose von einem bestehenden oder entstehenden Throm. bus herrühren kann.

Nun ist die Entstehung der Gehirnschädigung durch Embolie und das freie Intervall bzw. die Kenntnis ihrer Wechselwirkung an und für sich nichts Neues, wie auch Perthes angibt, es soll aber nach ihm, ,dieser Zusammenhang, der zuerst wohl von $\mathrm{Z}$ i m merma n $\mathrm{n}$ i 892 ausgesprochen wurde, dann mehr oder weniger in Vergessenheit geraten sein". Perthes führt mehrere solcher Fällc aus der Literatur an, die ich bier nicht zu wiederholen brauche. In Vergessenheit geraten ist diese Entstehungsart nun doch wohl nicht. $\mathrm{M}$ a $\mathrm{r} \mathrm{ch}$ and teilt unter anderm einen Fall mit, indem nach Rasiermesserschnitt mit leichtem Anschneiden der Communis nach dreitägigem Intervall der Tod durch multiple Embolien im Bereich der A. fossae Sylvii durch abgelöste Teile des (infektiösen) Carotisthrombus erfolgte (s. Artikel: Arterien in Eulenburgs Enzyklopädie). Zi e hen erwähnt diese Genese sogar in seinem Artikel über die internen Erkrankungen 
des Zentralnervensystems im „Handbuch der praktischen Medizin“ von Ebstein Schwalbe I 900 , indem er auf Embolien aus Carotisthromben nach stumpfer Gewalt hinwies. Pels-Le usden führt in seiner „Chir. Operationslehre" die ,fortschreitende Thrombose der Carotis communis nach ihrer Unterbindung bei Arterio. sklerose als wahrscheinliche" Todesursache an (S. 120). Ich selber habe mit Dietrich I9I7 denselben klinischen und anatomischen Befund erhoben, der ja bei beobachtetem freiem Intervall recht nahe liegt. I $c h$ meine also, da $B$ dieser $Z u$ sammenhang nicht vergessen wurde, sondern nur deshalb nicht häufig beschrieben wurde, weil er tatsächlich nicht allzu häufig angetroffen wird. sicher aber nicht in der Verallgemeinerung her. angezogen werden kann. wie Perthes es tut.

$P$ e $r$ the s eigener Fall, in dem die Exstirpation eines spontanen Aneurysma bei einer $44 \mathrm{j}$. Frau die Unterbindung der $\mathrm{Caro}$. tis interna dicht an der Schädelbasis nötig machte und in dem, nach 8 stündigem freicn Intervall nach 6 Tagen unter völliger Hemiplegie der Tod eintrat, ergab im intrakraniellen Teil der Carotis int und $\operatorname{der} \mathrm{A}$. cerebri ant. eine derbe Thrombusmasse. Es ist mir nun gar nicht „ganz klar", ob hier wirklich Embolie vorlag und nicht vielmehr ein Hineinwachsen des Thrombus aus der unterbundenen Interna: Die anfangs vorübergehende leichte Hemiparese und dann der zweite endgültige Nachschub lassen sich recht wohl durch Zuwachsen der wichtigen Gefäßäste erklären, was klinisch schubweise sich zeigen muB. Diese Erklärung scheint mir auch aus Gründen der Mechanik einfacher zu sein. Leider fehlen Angaben über ettwa vorhanden gewesene $\mathrm{mikroskopische} \mathrm{Infektion} \mathrm{an} \mathrm{der} \mathrm{Unter-}$ bindungsstelle. Die Unterbindung der Interna an der Schädelbasis ist überdies nicht gleichzusetzen mit den sonstigen verwcrteten Fällen der Unterbindung an der $\mathrm{Commun}$ is.

lch möchte alsodas Vorkommen wirklichen. bolischer Vorgänge nach Carotidenunterbindung sehr einschränken und eher die fortschreitende verlegende Thrombose gelten lassen, vor allem für solche Fälle, in denen ein freies Intervall beobachtet wurde (s. o.). Für die Mehrzahlder Fällevon Hirnstörung nach Carotidenunterbindung müsen wir doch auf andere Erklärungen zurückgreifen und ich weiß nicht. warum Perthes solche so wenig gelten läßt: „Einwandfreie 
Beobachtungen, die bewiesen, daß (iehirnerweichungen auch ohne voraufgegangene Thrombose oder Embolie der Gehirnarterie zustande gekommen ist, sind mir bis jetzt nicht bekannt geworden, während auf der anderen Seite die relative Häufigkeit der Fälle, in denen die Hirnstörung erst nach einem freien Intervall aufgegetreten ist, mit Bestimmtheit auf die große klinische Bedeutung des von uns hier betonten Entstehungsmodus hinweist."

Die erste Forderung der einwandsfreien "Beobachtung" nicht thrombischer Vorgänge könnte doch wohl nur an Verstorbenen erbracht werden, würde also für die Mehrzahl der Carotidenunterbindungen, die ja nicht in der Mehrzahl tödlich zu verlaufen pflegen, sondern nur mehr oder weniger schwere klinische Hirnstörungen zeigen, gar nicht erfüllt werden können. tber einerseits ist eine wirklich gefundene Throm. bose ander Leiche gar nicht mehrursächlich als Todesursache zu erweisen, nämlich dann nicht, wenn sler Gedanke an die oben als möglich hinngestellte prä a gona le 'T h rombose, wenn ich sie so nennnen darf, in gewissem Gegensatz zur agonalen oder postmortalen Gerinnung, durch den klinischen Verlauf und den pathologisch-anatomischen Befund nahegelegt wird. Und dann könnte ich bei einiger Hartnäckigkeit mit fast gleicher Berechtigung den Schluß ziehen, daß die Leute, die den Carotidenverschluß überstehen, nicht Thrombosen als Ursache ihrer vorübergehenden Hirnstörungen gehabt haben können, weil die Verstorbenen alle Thrombosen gehabt haben, diese also tödlich sein muB. Aber ich werde solchen Schluß nicht ziehen und lieber $P$ e $r$ the $s$ den Beweis zuschieben, da $B$ auch in den überlebenden schweren Fällen Gefäßthrombosen vorlagen und diese sich nicht aus anderen Gründen erklären ließen, und ferner die Darlegung, wie denn solche schweren Halbseitenlähmungen mit anatomischen Grundlagen dennoch und gar nicht selten spurlos ausheilen können? Ich komme selber später auf diesen Punkt zurück.

Die relative Häufigkeit des freien Intervalls aber muB ich auf $G r u n d m e i n e r e i g e n e n ~ B e o b a c h$ tungen und a uf Grund von Literaturstudien bestreiten. Dieses freie Intervall müßte aber doch klinisch folgerichtig bei allen Unterbindungen mit Hirnerscheinungen ge- 
fordert werden, wenn Thrombose oder Embolie angenommen wird, es dürfte nicht nur „relativ“ häufig sein!

Wohl zu bedenken ist ferner, daß der Tod bei Leuten, die einer Carotisunterbindung unterzogen wurden, doch auch gar nicht immer durch sie bedingt wird, sondern nicht selten durch Wundschlagkollaps, durch Blutungskollaps, durch Sepsis, Operationskollaps usw. und ein freies Intervall zwischen Operation und ersten Bewußtseinsstörungen usw. gar nicht immer mit der Carotisunterbindung und etwa nachher gefundener Thrombose zusammenzuhängen braucht.

Das freie Intervall wird uns noch öfters beschäftigen müssen, da es eben nicht einheitlich gedeutet werden kann.

Soweit die pathologisch-anatomischen Verhältnisse! Von anatomisch-physiologischenGesichtspunktenaus spielt die Unterbindung der verschiedenen Abschnitte der Carotis, der Wurzelarterie für das Gehirn, fürdieses eine verschiedene Rolle.

Perthes meint, „daß die tödlichen Hirnstörungen viel häufiger nach Unterbindung der Carotis communis als nach Unterbindung der Carotis interna beobachtet werden". Das ist durchaus nicht erwiesen, denn es sind die Unterbindungen der Interna nicht nur viel seltener ,veröffentlicht", sondern sicher auch sehr viel seltener ausgeführt, schon weil die Anzeige dazu seltener gegeben ist. Ich bin, wohl mit Pels-Leusden (l.c.) und S chmieden "Der chirurg. Operationskurs" 1920, vom Gegenteil überzeugt.

Die Ca rotis com. - ich darf zur Veranschaulichung des nicht visceralen Arteriensystems, abgesehen von den anatomischen Atlanten, auf die stereoskopischen Röntgenbilder $\mathrm{H}$. Hildebrands (s. „Das Arteriensystem" Fig. I u. 2 im Atlas stereoskopischer Röntgenbilder von Hildebrand, Scholz, Wie t ing) hinweisen - verläuft als dicker Stamm, in der Regel ohne Abgabe eines nennenswerten Astes, bis zur Gabelung in Carotis ext. und int. Die Externa beginnt nun sofort sich zu verzweigen und steht mit der anderseitigen Externa durch zahlreiche Verbindungsätze in Zusammenhang.

Nach Unterbindung der Communis kann durch Externaverbindungen die Auffüllung der noch 
offenen Interna auf der Seite der Unterbindung aufrecht erhalten werden und so das Gehirn, besonders wenn die Speisung durch den Circulus Willisii und die Vertebralis sowie die anderseitige Carotis interna funktioniert, leidlich genährt und in seiner Funktion aufrecht erhalten bleiben. Dies betont Perthe $\mathrm{s}$ besonders, um die Unzulänglichkeit der bisherigen Theorien über die Genese der Hirnstörungen zu erweisen.

Anders liegt die Sache bei der Carotis interna: sie setzt sich ohne Verzweigung aus der unverzweigten Communis fort bis zum Eintritt in die Schädelhöhle, um die wichtigsten Teile des Großhirns zu versorgen. Die geringfügigen A n a s to mos e $n$ ihrer Augenhöhlenäste mit denen der gleichseitigen Carotis externa sind für die Kollateralausbildung kaum von Bedeutung. sie genügen vielleicht, die Ophthalmicazufüllen, nicht aber das Großhirn nennenswert zu speisen. Nur die Verbindung der beiderseitigen A. A. cerebrales ant. durch die Rami communicantes aus dem Stromgebiet beider Internae, und in geringerem Grade die durch die beiderseitigen Rami communicantes post. aus den A. A. cerebri post. (Stromgebiet $\operatorname{der} A$. vertebralis, $A$. basilaris aus den beiderseitigen A. A. vertebrales) kann die Ernährung des Gehirns nach Unterbindung einer Carotis interna aufrecht erhalten, also Stromgebiete die auch nach der Unterbindung einer Communis unbeeinflußt bleiben würden.

Somit muB eine Sperre der Carotis interna, rein anatomisch gedacht. für das Gehirn sicher. lich verderblicher sein als eine solche der Caro. tis communis, bei welcher der Rückstrom aus der Carotis externa offen bleibt. Und noch ungünstiger werden a prioridie Bedingungen fürdas Gehirn, wenn Ca. rotis externa und interna (oder auch communis) isoliert unterbunden werden.

Zahlenmäßige Belege für die Gefahren der jeweiligen Un terbindung zu bringen, hält nachträglich schwer, da die Beobach. tungen nicht alle eindeutig sind und manche Punkte, besonders auch der der Infektion, wenig berücksichtigt sind. Wenn Pilz, wie viel zitiert wird, 1868 unter 520 älteren Fällen von Ligatur der Carotis communis in 165 Fällen klinisch Hirnerscheinungen 
verzeichnet mit $9 £$ Todesfällen, so sagt das weniger als die kleinere aber wenigstens eine einheitliche Indikation aufweisende Zusam. menstellung Beckers, der 72 Fälle von pulsierendem Ex o p h th a I $\mathrm{m} \mathrm{u} \mathrm{s}^{1}$ ) verzeichnet, die durch Unterbindung der Carotis communis (einfach und ohne Infektion!) behandelt wurden mit einer Mortalität von nur 3 Fällen, die der Operation zur Last $2 u$ legen waren. Für die Internaunterbindung habe ich keine gröBere Zusammenstellung gefunden.

Die feineren Zirkulationsverhältnisse des Ge. $\mathrm{h}$ irns sind von $\mathrm{M}$ a r chand kritisch besprochen (s. Marchand. Kre h l, Handb. d. allgem. Path., Artikel: Die Störungen der Blutver. teilung. Bd. Il. r). „Theoretische Erörterungen über die Zirkulationsstörungen (Druckschwankung, arterielle und venöse Hyperämie und Gefäßdilatation, seröse Durchtränkung, Liquorschwankung und anderes mehr) ohne Berücksichtigung der jeweiligen pathologisch-anatomischen Verhältnisse lassen sich nun teilweise auf die menschliche Pathologie anwenden. Die Annahme von der relativen Unveränderlichkeit einer Arterienstrecke bei experimentellen Versuchen über die Hirnzirkulation beruhen auf recht unsicherer Grundlage. Die Gehirnarterien sind ebensowenig wie die anderen Körperteile einem nur mechanisch veränderlichen oder gar unveränderlichen Röhrensystem gleichzusetzen. Für die embolischen und thrombo. tischen Verschlüsse der Gehirnarterien selber ist, ,ihr Charakter als Endarterien im Sinne Cohnheims, wobei kleine arterielle Anastomosen mit Nachbargefäßen sich nicht als ausreichend für die $\mathrm{H}$ erstellung der Zirkulation erweisen, wichtig; bei solchen tritt in den meisten Fällen peripherisch vom Verschluß zunächst Stillstand des Blutes in den Arterien wie den zugehörigen Venen ein. Es hängt sodann hauptsächlich von der Anordnung des Kapillarnetzes $a b$, ob das abgesperrte Gebiet von den noch offenen Nachbarästen aus hinreichend Blut zugeführt werden kann oder nicht. Die größeren von den basalen HauptgefäBen abgehenden Stämme (A. A. cerebri ant., med. und post.) besitzen außer dem Circulus Willisii keine kollateralen Verbindungen (Heubner, Duret). Die Endausbreitungen der Hauptarterienäste in der $\mathrm{Pia} \mathrm{m}$ at $\mathrm{er}$ hingegen (Ramicorticales n. Cornet) bilden an der Oberfläche des Gehirns ein dichtes Netzwerk von kommunizierenden Arterien, so daß es leicht gelingt, von einem Stamm aus große Nachbargebiete mit Injektionsmasse zu füllen. Die kleinen von diesen oberflächlichen Stämmchen in die Hirnsubstanz eintretenden Arterien haben jedoch keine kollateralen arteriellen Verbindungen untereinander, sondern sind End-

1) S. a. den Artikel C. H. Sattlers in v. Graefe.Sae mischs Handb. d. gesamten Augenheilkunde 1920 „Pulsierender Exophthalmus". 
arterien, die sich in Kapillaren auflösen. Auch die größeren von der Basis aus in die großen Hirnganglien eintretenden Stämme ( $\mathrm{H}$ e u b ners Basalbezirk), die Rami basales n. Cornet, haben keine arteriellen Verbindungen untereinander und stehen auch mit den Gefäßen der Pia mater höchstens in einzelnen Ästchen in Verbindung. Da somit die kollaterale Blutversorgung sich zunächst nur auf die Randpartien beschränkt und die Substanz des Zentralnervensystems nur sehr kurze Zeit die völlige Absperrung der Sauerstoffzufuhr verträgt, so stirbt sie ab, bevor sich eine kollaterale Zirkulation ausbilden kann.Wir müssen diese Darstellung $M$ a $r c h$ and s wohl als die zutreffende bezeichnen, die durch H e u bners Angaben, daB sich zwischen dem Netzwerk der Pia mater Verbindungen bis zu I mm Stärke finden, bekräftigt werden; während andere, namentlich Cornet (s. sein Lehrbuch der topographischen Anatomie 1917) die drei großen Hirnarterien (A. A. cerebri ant., med. und post.) und ihre Rami corticales als Endarterien bezeichnen, so daß die vorhandenen Anastomosen nicht genügen würden zur Versorgung des ausgeschalteten Gebiets.

Diese kurze Wiedergabe der einschlägigen feineren anato. mischen Beziehungen scheint mir deshalb von Wichtigkeit, weil sie für die Frage entscheidend sein dürfte: welche Art von Embolie -- wenn man eben für alle Fälle eine anatomische Grundlage finden will - denn nötig ist, das Leben $z u$ gef ährden? In Perthes anatomisch untersuchtem Falle findet sich ein Embolus (oder sekundärer Thrombus?) aus der Un. terbindungsstelle im intrakraniellen Teil der Carotis interna, da, wo dic A. cerebri ant. abging; es ist nicht gesagt, wie weit dieser Thrombus ging, z. B. ob auch der Ramus communic. ant. und der normale starke Ast oberhalb des Balkenknies zur rechten Seite ebenfalls geschlossen war. Wenn die Carotis interna am Abgang der A. cerebri ant. geschlossen ist, dann muß auch die A. cerebri med. A. fossae Sylvii, als eigentliche Fortsetzung der Carotis cerebralis, verschlossen sein, so daß wir sagen können, wir haben es in dies e $\mathrm{m}$ Falle mit einer recht ungünstigen Kombination zu tun, in dem die Verhältnisse des Circulus Willisii gar nicht in Betracht kommen. Wäre allein dic A. cerebri ant. oder die medialen oder basalen Äste derselben embolisiert, so würde wohl kaum in jedem Falle ein so ungünstiger Verlauf eintreten. Kennen wir doch a poplektische Insulte 2. B. bei Atherosklerotikern mit anfangs recht schwerem Verlauf, der dann doch schließlich über eine Hemiplegie in wesentliche Besserung übergehen kann. Wir wissen ja bei diesen apoplektischen Insulten 
auch nur selten im voraus, welcher zum Tode führt und welcher einer Besserung fähig ist, und für die tödlich verlaufenden Fälle finden wir durchaus nicht immer eine a $\mathrm{n}$ a to $\mathrm{m}$ is $\mathrm{ch}$ hinreichende Erklärung, müssen uns vielmehr oft genug damit begnügen zu sagen, daß der apoplektische Insult in dem betreffenden Falle eben hinreichte, dem Leben ein $\mathrm{Ziel}$ zu setzen, ohne dasselbe Schicksal in einem anderen ähnlichen Falle voraussagen $z$ u können. Wir wären somit auch klinisch zu dem angedeuteten Ergebnis gekommen, daß $\mathrm{klin} \mathrm{i-}$ sches Bild und rein anatomischer Befund nicht immer sich vollständig decken, es gehören dazu noch ein funktioneller oder biologischer Faktor.

Es dürfte wohl verlangt werden, daB, wenn man eine Theorie der traumatischen Embolie nach Carotisunterbindung $\mathrm{zu}$ ihrem Rechte bringen will, diese in Einklang gebracht werde mit den bisherigen klinischen Erfahrungen über hirnembolische Vorgänge und diese besitzen wir ja in ausreichendem Maße in der inneren Medizin, besonders nach endokardischer oder endarteriitischer Thrombenbildung, welchen Ursprungs auch immer. Ihre Erscheinungen, die nicht einmal immer von hämorrhagischer Apoplexie zu unterscheiden sind. hängen wohl von dem Sitz des verschließenden Embolus ab, sind aber, wie gesagt, nicht so eindeutig wie man annehmen sollte, es spielt da die Wertigkeit des Endorgans und gewisse funktionelle Schädigungen eine nicht unwesentliche Rolle. Nach Ziehens oben zitierter Zusammenstellung erzeugt die Embolie der Carotis interna „bald relativ leichte, bald sehr schwere Symptome; in schweren Fällen wird die Funktion bald der ganzen zugehörigen Hemisphäre ausgeschaltet und meist kommt es dann zu einem rasch töd. lichen Ausgang". Die Schwere der Erscheinungen hängt nach $Z$ iehen $a b$,von der eventuellen $A$ usbildung des sekundären Thrombus, und je nachdem der Circulus Willisii dank seiner individuellen Anlage und dank der individuellen Leistungsfähigkeit des Herzens eine vikariierende Blutversorgung herbeiführt oder nicht". Der Insult bei Embolie der A. cerebri m ed. ist, abgesehen von den örtlichen Herdsymptomen gekreuzter Hemiplegie ,überhaupt nicht erheblich“. Strittig ist nach $Z$ i e h e $n$ 
roch die Erklärung der Bewußtseinsstörung nach Hirnembolie. ,Wernicke betont das traumatische Moment; Marchand hält den plötzlichen Funktionsausfall eines großen Teils einer Hemisphäre für wesentlich, $\mathrm{M}$ on a kow nimmt an, daß eine reflek. torische Erregung der vasomotorischen Zentren mit konsekutiver Hirnanämie eintritt; meines ( $\mathrm{Z}$ i ehens) Erachtens spielt die plötzliche Hirndruckschwankung die Hauptrolle."

Ich meine nun, daß wir mit der anscheinend so sicher begründeten Embolietheorie nach Carotisunterbindung auf Grund der Vergleiche mit der Erfahrung aus der inneren Medizingar nicht viel gewonnen hätten, wir hätten das Themanur etwas weiter cerebralwärts verschoben! Und ich meine, daß die gewaltige Strom-und Druckveränderung, dieaugenblicklicheintretendeschwere Ernährungs-bzw. Funktionsstörung, wiesiedurch den operativen VerschluB einer so groBen und wichtigen Strombahn der Carotis communis oder Interna geschaffen wird, eine höhere Bewertung fürdie Erklärungim physiologischfunktionellen Sinne verlangt als die Embolie kleinerer Gehirngebiete, deren Untergang ja nicht das Leben $z \mathrm{u}$ vernichten braucht. Das beweisen uns die cerebralen einseitigen apoplektischen Blutungen, die oft bedeutenden örtlichen Zerstörungen von Gehirnsubstanz durch Schußverletzungen ohne tödlichen Ausgang, denen eben das Moment der gewaltigen Blutzufuhrschwankung fehlt.

Liegt es nun schon ganz außerhalb unserer genauen Kenntnis, wie im norma len Gehirn die Strömungsverhältnisse in dem verwickelten Netz der Basalarterien und ihren Zweigen liegen, welche Richtung z. B. im Circulus Willisii jeweilen der Blutstrom nimmt, und müssen wir annehmen, $\mathrm{da} \beta$ schon normalerweise in diesem Gefäßnetz wie auch in der so vielfach gewundenen Carotis interna selbst Stromwirbel und Stromverlangsamungen bis zum vorübergehenden örtlichen Stillstand durch Gegenströmungen sich bilden müssen, so ist es wohl ziemlich unmöglich, sich ein Bild davon zu machen, wie es z. B. unter teilweiser Ausschaltung eines Stromgebietes wie es der Carotis communis und 
eventuellen Ersatz durch die Externaäste gestalten würde. Ich möchte demnach zwar nicht von der Hand weisen, daß besonders gut ausgebildete anatomisch vorhandene Kollateralen der Aufrechterhaltung oder Wiederherstellung der Blutversorgung für das Gehirn günstig sind, halte aber Schlußfolgerungen alle in aus dem, was man bei der Nekropsie findet, also zumeist in Fällen, in denen die Kollateralen nicht ausreichten, trotz normaler $\mathrm{Ge}$ fäßverhältnisse, für nicht beweisend weder im positiven noch im negativen Sinne.

Man kann nicht die physiologischen Funktionen eines GefäBbaumes allein aus seinem anatomischen Bau ableiten; man muß sein physiologisches und pathologisches Arbeiten auch mit der physiologischen und pathologischen Tätigkeit seines Endorgans in Einklang bringen und diese wird letzten Endes sich nur durch die klinischen Erscheinungen kundgeben. Das will sagen: wir können für das Darniederliegen oder gänzliche VersagenderGehirnfunktiongarnichtimmereine anatomische Grundlage, auch nichtander Leiche erwarten.

Auch in anderen Stromgebieten finden wir ja häufig genug ganz verschiedene Wirkungen auf das Endorgan nach Sperrung eines Gefäßes, ohne jedesmal eine anatomische Unterlage dafür erwarten $z u$ dürfen. Das ist bekannt $z$. B. für die Unterbindung der A. femoralis in ihren verschiedenen Abschnitten. Bekannt vor allem ist die Wirkung der Sperre der A. poplitea, die so sehr oft zur Gangrän der peripheren Teile führt, ohne daß ein Embolus oder eine absteigende Thrombose vorhanden wäre, und ohne daß man eine Ursache dafür wüßte, warum in den meisten Fällen Gangrän eintritt, in vielen aber ausbleibt: es bleibt dann eben nur dic Annahme, daß eben der Kollateralkreislauf, sei es nun aus anatomischer oder aus funktioneller Ursache, im Ver $\mathrm{h}$ äl $\mathrm{t} n$ is zur Vitalität des von ihm zu versorgenden Gebietes nicht a usreichte. Ein Blick auf das Bild 9 und ro des Hildebrandschen stereoskopischen Atlas bringt uns die anatomischen Verhältnisse am Unterschenkel nahe. Würde man überall nach Carotisverschluß von einem größeren offenen Gehirngefä $B$ aus die betrnffene Hirnhälfte mit In jektion smas se füllen und stereoskopisch die Kollateralkreislaufbedingungen klar- 
legen, so würde sich wohl manches aufklären lassen, was die einfache Autopsie nicht zeigt, dennoch aber dürften wir auch ein solches Ergebnis nicht als beweisend ansehen für die Lage, die intra vitam wirklich geherrscht hat: $G$ efä $B$ kontraktion en, die bestanden haben können, lösen sich durch die In jektionsmasse, nur anatomisch vorhandene Verschlüsse kommen zur Anschauung, ein Hinweis, der aucl für andere Stromgebiete gilt, z. B. die Nierenpathologie zur Scheidung zwischen funktionellen und anatomischen Störungen. Bezüglich der angiosklero. tischen Gangrän wies ich schon vor Jahren auf diese Dinge hin.

Und die für das Gehirn in Frage kommenden physiologischen und biologischen Verhältnisse sind gar nicht so leicht wiegend, wie Perthes sie wiegt. Die zentralen Abschnitte des Nerven. systems haben einen lebhafteren Stoffwechsel als die peripheren Teile. Gehirn und Rückenmark leiden daher bei Behinderung der Blutzufuhr und Störung der Ernährung viel schneller als die peripheren Nerven (s. Landois-Rosemann, Lehrbuch der Physiologie 1917). Nach Marchands zusammenfassender Darstellung (l.c.) ,verträgt die Substanz des Zentral. nervensystems nur sehr kurze Zeit die vollstän. dige Absperrung der Sauerstoff $z$ ufuhr", sie recimet mit Minuten bis kaum einer Stunde, je nach dem Grade der Plötzlichkeit der Absperrung und nach dem Zustand des Gehirns bzw. Rückenmarks.

Klinisch spielt die zeitliche Abhängigkcit der Gehirnfunktionsstörung von dem Akt der Blutsperre eine wichtige Rolle; da das Gehirn, wie gesagt, nur ganz kurze Zeit die voll. kommene Blutsperre verträgt und seine Substanz, namentlich die graue, früh abstirbt, müßte nach einer endgült gen Sperre eine Erholung ausgeschlossen sein, die Hirnerschein ungensich also unmittelbaran eine etwaige operative Sperre anschlieBen, falls sie auf diese zurückzuführen sind. Da nun aber die wirkliche Zeitdauer und die Vollständigkeit der Sperre wie auch die physiologische Wertigkeit des Gehirns und seines Gefäßsystems nicht festliegende Momente sind, werden auch die klinischen Erscheinungen in ihrer Schwere nicht konstanl sein.

Ich darf aber feststellen, daß die klinischen $B$ eobach. 
tungen namentlich bezüglich der Zeitfolge von Operation und Hirnsymptomen indenallermeisten Fällen recht ungenau sind: Vielfach, namentlich zu Zeiten starker Inanspruchnahme oder zur Nachtzeit, gestaltete sich die Sache doch so, daß die mehr oder weniger mit Morphium oder Scopolamin eingedämmerten Kranken in Narkose operiert und nach der Operation halb wach ins Bett gebracht werden, wo sie dann allenfalls unter den Augen des Pflegepersonals ihre Narkose ausschlafen, nicht selten zur ewigen Ruhe. Wer will dann sagen, ob und wenn z. B. eine Halbseitenlähmung, ob überhaupt Bewußlosigkeit als Folge der Hirnzirkulationsstörung bestand, ob ein freies Intervall vorlag u.a.m.? B. Kohn teilt in seiner „Klinik der embolischen Gefäßkrankheiten" (Berlin 1860) einen Fall mit, in dem bei einem durch Blutverluste schon sehr anämischen Mädchen nach Unterbindung der einen Carotis communis sofort eine halbseitige Lähmung der Extremitäten eintrat, nach der sofortigen Lösung der Ligatur aber schwanden die Lähmungen und das Eewußtsein kehrte zurück. Hier ist der experimentelle Beweis am Menschen erbracht einmal dafür, daB die Blutsperrung einer Carotis com. munis allein sofortige schwerste Hirnstörungen herbeizuführen vermag, und zweitens der Beweis erbracht, daBdie Wirkung der Blutsperreauf das Gehirn eine fast augenblickliche sein kann, daß sie aber in diesem Stadium, nach kurzer Zeit, als funktionell bedingt, noch des völligen Ausgleichs fähig ist.

Es ist mehrfach die Bemerkung gemacht worden, daß die Gründe für Gangrän der Nekrosen im Endorgan im wesentlichen von dem Verhalten der Kollateralen zu suchen seien ( $G$ u in a $r d$, Ed. Rehn). Natürlich die Armut kommt von der pauvretél Erhielten die Endorgane nicht zu wenig Blut, würden sie auch nicht blutarm werden. Warum aber erhalten sie zu wenig Blut, zu wenig. um weiterleben zu können? Das ist die Frage des Seins oder Nichtseins für sie!

Es ergeben sich nun für die Praxis folgende physiolo$\mathrm{g}$ is ch biologis chen Momente, die neben den anatomischen Veränderungen vollster Würdigung fürdie Erklärung 
der Hirnstörungen nach Carotidenunterbindung verlangen.

Erstens: Besteht im Augenblick der Carotisunterbindung eine allgemeine Anämie, etwa durch Ausblutung, so wird durch sie die örtliche des Gehirns naturgemä $B$ vermehrt und die oft schon tief durch den Blutverlust herabgestimmte Funktion ganz aufgehoben werden. Die allgemeine Anämie genügt allein schon, eine Carotisunterbindung tödlich zu gestalten, die derselbe, nicht anämische Mann vielleicht überstanden hätte (s. Fall 6).

$Z$ weitens: DurchflieBt den Körper und somit das Gehirn im Augenblick der Unterbindung einer Carotis minderwertiges Blut, das den Gas- und Stoffwechsel nur ungenügend vermittelt, und haben die nervösen Parenchymzellen durch die gleiche Schädlichkeit wie das Blut in ihrer Wertigkeit schon gelitten, so wird eine weitere Blutsperre für das Gehirn, und sei sie auch nicht vollständig, ganz besonders zerstörend wirken. Solche Vorbedingungen sind praktisch gegeben namentlich bei $\mathrm{s} c \mathrm{~h}$ weren p yog e ne n oder toxischen Allgemeininfektionen, wenn z. B. eine gleichzeitige Halsgefäßverletzung eine solche Gefäßunterbindung dringend machte. $\mathrm{Zu}$ der schweren Veränderung des Blutes, das wässerig lackfarben werden kann, kommt die durch manche Infektion bedingte vermehrte Neigung des Blutes zur Thrombenbildung (s. Beneke, l.c.). „Angeborene Ge w.ebseigentümlichkeit, bestehend in erhöhter Gerinnbarkeit des Blutes oder sie fördernde Sekretion des Gefäßendothels annehmen zu müssen", wie Stierlin und v. Meyenburg tun, werden wir nicht selten Anlaß haben.

Drittens: Die gute Blutversorgung des Gehirns, die Eudihämorrhesis, hängt nicht nur von der Blutbeschaffenheit $a b$, sondern auch von dem $Z$ ustand der Gefä $B$ e. In dem so hochwertigen Parenchym mit seinem großen Gefäßreichtum und seinem komplizierten Lymph- und Liquorsystem muß ein rascher Ausgleich zwischen Gefäßfüllung und Liquor gewährleistet sein, soll das Gehirn dauernd vollwertig funktionieren. Je weniger anatomisch und physiologisch die Gefäßwandungen in Muskulatur, Endothel, Eigenernährung und Innervation der Norm entsprechen, desto eher wird eine hinzutretende Schädigung das Gleichgewicht 
stören. Auch hier spielen die sog. chirurgis chen Infek tionskrankheiten eine wesentliche Rolle, indem die Gefäßwandungen toxisch sowohl zellulär wie funktionell geschädigt werden. Andere Infektionskrankheiten, wie z. B. das so sehr das Gefäßsystem angreifende Fleckfieber, können auch einmal in Betracht kommen, ebenso wie Intoxikationen durch Kriegsoder Nahrungsschäden. Namentlich aber ist hier das Alter in Rechnung zu setzen: das alternde Gehirn ist nicht allein durch die grob anatomisch sklerotischen Veränderungen der Gefäßwandungen und die dadurch herabgeminderte Befähigung zur Schaffung eines ausreichenden Kollateralkreislaufes minderwertig, sondern ist es auch durch die verminderte Anpassungsfähigkeit der Parenchymzellen der grauen und weißen Substanz: es ist also darum schon durch eine Carotisunterbindung viel leichter zu schädigen als ein jugendliches, anpassungsfähiges Gehirn. Dies ist und bleibt der Grund für die bekannte Empfindlichkeit des alternden Gehirns gegenüber einer Carotissperre, nicht so sehr aber die von Perthes in den Vordergrund geschobene Anschauung, „da $\mathrm{B}$ infolge der Brüchigkeit des Gefäßes (der Carotis) die Vorbedingungen für die Thrombose an der Unterbindungsstelle in besonderem Maße gegeben sind". Th. Kochers Ausspruch: ,Man mag sagen was man will, die Unterbindung einer Carotis communis bei einem alten Mannmit Arteriosklerose ist einem Todesurteile gleichzusetzenl" besteht nach wie vor zu Recht.

In den drei vorstehend verzeichneten Verhältnissen spielt als Teil des Gefäßapparates auch die Pumpzentrale, das Herz eine wichtige Rolle: sein eigener Zustand, beeinflußt durch einen jener 3 Faktoren oder unabhängig von diesen aus diesem oder jenem Grunde zu Minderleistung verdammt oder zur Mehrleistung befähigt, kann den Ausschlag für das Schicksal der herzfernen Organe geben.

Viertens: Aus der Gefäßchirurgie ist bekannt, daß mechanische, thermische und andere Reize bei Manipulationen an den Gefäßen namentlich den Arterien spastische Kontraktion der $G$ ef ä $B$ wandung hervorrufen kann. Ich wies früher auf diese Tatsache hin, daß während der Operation, der bloßen Freilegung einer Femoralarterie, sich die mechanisch gereizte Arterie 
bis zum völligen Aufhören der Pulsation und Verwandlung des Gefäßes in ein starres enges Rohr spastisch zusammenziehen kann und nahm damals als Grund'age für diese Erscheinung die a $\mathrm{n}$ g io spastische Veranlagung der betreffenden Gefäße an, zumal a u ch die Ven en dasselbe Bild zeigen können (s.W i et ing, „Die angiosklerotische Gangrän usw.“. Deutsche Zeitschr. f. Chir. 1911, Bd. I1o und I9I2, Bd. I19). Eine ähnliche Erklärung bil. dete ich mir für einzelne Erscheinungen bei Verschütteten in diesem Weltkriege und L. Frankenthal gab demselben Gedanken dann Ausdruck in seiner Abhandlung ,,Über Verschüttungen“"s. Virchows 'Archiv 1916, Bd.222, in der er sagt: „Es muß aber auch damit gerechnet werden, daß eine so hochgradige Kontraktion der Gefäßwand (bedingt durch eine reflektorische Wirkung auf die Vasokonstriktoren) bestand, daß daraus allein schon die ischämische Nekrose resultierte." Diese Beobachtungen erfuhren nun eine außerordentlich interessante $\mathrm{Er}$. gänzung durch die von $K \ddot{u} t \mathrm{tne} r$ und $B$ a ruch jüngst mitgeteilten Befunde des von ihnen treffend bezeichneten und als solchen einwandsfrei verfolgten „s egmentären Gefä $B$. krampfes": sie fanden mit anderen, namentlich Kroh (Bruns" Beiträge z. klin. Chir. 1915, Bd. 97 und 1917, Bd. 108) in das Arterienrohr nahe streifenden, es aber nicht zerreißenden $S \mathrm{ch}$ u $B$. wunden eine rein örtliche Gefäßkontraktion, die, mindestens mehrere Stunden bestehend, einer Sperrefast gleich kommen; sie definieren das Krankheitsbild als ,mehr oder weniger vollständige Aufhebung der Zirkulation eines Gliedes nach traumatischer Irritation der Gegend der

A nme r kung: So interessant es auch ist, daß a u ch die Franzosen ähnliche Krankheitsbilder z. B. unter dem Namen ,s'upeur artérielle" in diesem Kriege beschrieben, brauchen wir ihnen doch n.cht bescheiden die Priorität lassen, zumal wir in diesen Ze:ten unsere wisssenschaftliche Arbeit besonders schützen und stärken mïssen, wo wir nur können; wir brauchen sie ihnen deshalb nicht zu lassen, weil sowohl patho-physiologisch (s. Marc ha n d - K rehl) ähnliches bekannt war und weil klinisch auch von $\mathrm{me}$ in e $\mathrm{n}$ über Frankenthals und $\mathrm{Krohs}$ bis zu Küttners Feststellungen eine fortlaufende Entwicklung dcsselben Gedankens festzusiellen ist. Ubrig ns hat Pirogoff $\mathbf{1} 866$ bereits dieselbe Er cheinung an $\mathrm{d} \mathbf{r}$ unvelletzten Arterie, wenigstens klinisch, genau beshr.eben! Grundzüge d.r allgemeinen Kriegschirurgie, S. 249. 
versorgenden Arterie, meist ohne eigentliche Verletzung der Gefäßwand" (s. Bruns' Beiträge z. klin. Chir. I920, Bd. I20). Daß gleiche Zustände a uch an den Gehirngfä $B$ en vorkommen können, beweist die von $\mathrm{Küt} \mathrm{tner}-\mathrm{Baruch}$ wiedergegebene Beobachtung 8 , in der ein segmentärer GefäBkrampf der Carotis communis mit hemikranischen Beschwerden gefunden wurde, doch war das Gefäßrohr nicht völlig zusammengekrampft und der periphere Teil füllte sich offenbar von der anderseitigen Carotis externa her, so daß keine völlige Blutsperre eintrat.

F ünftens: Von diesem stundenlang anhaltenden segmentären Verschluß der traumatisch geschädigten Schlagader, der vielleicht gar nicht so selten ist, dann aber, wenn er stundenlang dauerte und vollständig war, wohl, da seine Folgen augenblicklich eintreten müssen, das Leben häufig $\mathrm{ras} \mathrm{ch}$ beendet haben wird oder doch mit den schwersten, meist irreparablen Hirnstörungen eingeliefert werden dürfte, von diesem Verschluß also wohl $z u$ unterscheiden ist die Kontraktion des ganzen peripheren GefäBabschnitts jenseitseiner Unterbindung, auf die Marchand namentlich hinweist. Perthes lehnt zwar die Möglichkeit einer Hirnstörung durch Gefäßkrampf ab, ,wenn das richtig wäre, sollte jede rasche Unterbindung der Carotis diese Folgen haben, was nicht der Fall ist". Es ist aber die Dauer dieser Gefäßkontraktion nicht genügend bekannt und es ist wohl - in Einklang mit dem im vorstehenden Abschnitt erwähnten segmentären Spasmus, weshalb ich etwas näher auf diesen einging - wohl verständlich, daß diese Dauer bei den einzelnen Individuen recht verschieden sein wird, je nach dem allgemeinen oder auch örtlichen Reizzustand, der gerade im Gefäßgebiet herrscht und somit in dem einen Falle für die Ernährung des Gehirns von Bedeutung, in einem anderen ungefährlich sein wird. Ich erinnere hier an die bei Ausgebluteten eintretende allgemeine spastische Verengerung der Arterien, die selbst größere Gefäße vom Kaliber der Radialis auf Stunden ganz schließen kann, und erinnere an die sog. spontane Blutstillung selbst größerer Gefäße wie der Femoralis bei Abrissen oder Abschüssen ganzer Glieder, die nicht zum geringen Teile auf langdauernden, allerdings vielleicht örtlich 
„segmentären" Gefäßkrampf beruhen dürfte. Wenn Mar chand (1.c. „Der Kollateralkreislauf“) sagt: „Wird eine größere Arterie mit Kollateralen in der Kontinuität unterbunden, so füllt sie sich in der Regel sehr schnell, ja unmittelbar durch die vorhandenen peripheren Anastomosen in umgekehrter Stromrichtung; die Füllung erfolgt um so schneller, je dehnbarer die Arterien sind, also bei jugendlichen Individuen", so ist dabei wohl zu verstehen, daß Gefä $B$ füllung und Herstellung des Kollateralkreislaufes nicht dasselbe ist, und daß es mit beiden gerade bei der Carotis, besonders der Carotis in terna, wie oben ausgeführt, recht schlecht bestellt ist. Marchand schildert denn auch die Entstehung des a nämi. schen Infarkts im Gelirn nach Arteriensperre recht anschaulich.

Sechstens: Die Erhöhung des Blutdrucks, die Per. the $s$ aus A. Biers Versuchen über „Die Entstehung des Kollateralkreislaufs" (s. Virchows Archiv I 898, Bd. I 47 u. I 53) ableitet, als eintretend nach Unterbindung der Carotis communis und als bedeutungsvoll für das rasche Eintreten des rückläufigen Blutstroms aus der Carotis externa einschätzt, kommt für die Ausbildung eines wirklichen Kreislaufes nicht in Frage; peripher von einer Unterbindungsstelle sinkt vielmehr der Blutdruck nach Unterbindung in den peri. pheren Carotisabschnitten und solche Herabsetzung der peripheren Widerstände ist der Ausbildung der Kollateralen eher günstig als die Erhöhung des Blutdrucks!

Siebentes: Lag schon zum Teil in den vorstehenden sechs Punkten enthalten, daß einzelne nur für besondere vorliegende Verhältnisse in Frage kommen, so liegt auf der Hand, $\mathrm{da}$ natürlich die Operationsanzeige von wesent. lichem Einflu $B$ auf Art des Eingriffes und Verlaufes wie Folgen des geschaffenen Zustandes sein müssen. Sei es die bestehende Erkrankung, z. B. Tumor, Aneurysma, die vorher schon gewisse Veränderungen, wie langsame Kompression oder Drosselung, aber auch Thrombenbildung geschaffen haben kann, sei es die Art des Eingriffes, oder je nach der Anzeige eine plötz liche Sperre zum Gehirn erfordert wie bei Verletzungen mit Blutungserscheinungen, oder eine langsame Drosselung wie bei $\mathrm{Tu}$ - 
moren, Epilepsie oder Inzisionsneuralgie u. a. m., immer sind diese Beziehungen zu berücksichtigen, d. h. wir können die Ge. $\mathrm{f} \ddot{\beta} \mathrm{sperrungen} \mathrm{nicht}$ alle über einen $\mathrm{Kamm} \mathrm{sche-}$ ren weder vom technisch operativen noch vom prognostischen Gesichtspunkte aus.

Gesteigerte funktionelle Anforderungen und funktioneller Reiz ( $\mathrm{R}$ o u x), Blutgefühl der Gewebe (A. B i e r) fordern die Anpassung der Gefäßversorgung und ihre nervöse Regulierung durch Dilatation der in Frage kommenden Gefäßbezirke mit Erweiterung und Verstärkung der Gefäße, lösen die Anpassung aus. Es sind jedenfalls die Verhältnisse bei der Ausbildung des Kollateralkreislaufes recht verwickelt (s. Marchand 1.c.) und zeitlich wie qualitativ nicht abzumessen. Auf solche, nicht auf die rein mechanischen Blutdrucksteigerungen müssen wir zurückgreifen zur Erklärung, weshalb, namentlich bei Jugendlichen, so häufig weder nach Unterbindung der Com. munis noch besonders nach der der Interna der Tod oder doch schwere Störungen der Hirntätigkeit eintreten und auch dafür, warum eingetretene schwere Störungen selbst nach langer Zeit sich noch völlig ausgleichen können?

Diese letztere Frage erheischt angesichts der Tatsache, daß das Gehirn nur ganz kurze Zeit die völlige Absperrung der Sauerstoffzufuhr verträgt, ganz besonderes Interesse und bedarf der Erklärung. Wenn wir von der Annahme absehen, daß die gesunde Hemisphäre vikariierend eintrete, einer Annahme, die angesichts des oft großen Ausfalls einseitiger Zentren und Bahnen wohl abzulehnen ist, können wir nur annehmen, da $B$ in $\mathrm{den}$ überlebenden Fällen eben immer doch keine so tiefe Zerstörung der Hirnsubstanz vorlag, daß trotz schwersten Darniederliegens der Hirntätigkeit, trotz tiefster Bewußtlosigkeit, in dem betroffenen Gebiet doch noch eine schwache Blutzufuhrdie Vitaminimades Gewebes a ufrecht erhielt: wir könnten an Ödemisierung bzw. Hirnschwellung denken als Grundlage der schweren Funktionsstörung, wie wir Gleiches ja durch die Untersuchungen von $\mathrm{Sch}$ ma us („Die Kompressionsmyelitis bei Caries der Wirbelsäule“ 1899) für das Rückenmark kennen, bei der auch eine völlige Wieder- 
herstellung möglich ist. Doch ich möchte hier auf diese Dinge nicht weiter eingehen und verweise besonders auf die $\mathrm{Erfah}$ rungenderinneren Medizin nach hirnembolischen Vorgängen und deren Reparation (s. auch Marchand 1.c. „Die Zirkulation im Gehirn" S.222).

Ich muB also dabei bleiben, dab die Unterbindung sowohl der Carotis communis als auch be. sonders die der Carotis interna fürdas Gehirnal. lein durch die plötzliche gewaltige Schwankung der Blutzufuhr, ohne jede sonstige anatomische Komplikation wie Embolie oder Thrombose für das Gehirn von schwersten Folgen begleitet sein $k a n n$, wenn a uch nicht $m u B$. Und für diese gewaltige augenblicklich eintretende Beeinflussung der Zirkulationsverhältnisse in der Schädelkapsel also auch im Gehirn, scheint mir die günstige therapeutischeBeeinflussung geradedes pulsierenden Exophthalmus, des arterio-venösen Aneurysmas im Sinus cavernosus, bedeutungsvoll zu sein. Das Aufhören der Pulsation im Augenblick der Unterbindung, das Rücksinken des Bulbus und in günstigen Fällen die endgültige Heilung dieser in das Gebiet der Carotis cerebralis einge. schalteten Blutgeschwulst beweisen doch den tiefgreifenden EinfluB der Carotisunterbindung a uf die Gehirnzirkulation, die also selbst dann eintritt, wenn eigentliche Hirnstörungen nach ihrer Unterbindung ausbleiben. In den günstig verlaufenden Fällen wird auch Perthes wohl keine Thrombose oder Embolie an der Unterbindungsstelle der Communis annehmen, und diese Wirkung kommt zustande, trotzdem die Kommunikation zwischen Interna und Externa aufrecht erhalten blieb, eine Verbindung, die ,als Ausgleichsvorrichtung zur Speisung der Carotis interna und des Gehirns ausreichen sollte"! Diese Unterbindung der Carotis communis wegen pulsierenden Exophthalmus hat nach Becker in 72 Fällen nur 3 mal zum Tode geführt; es wurde wohl in allen Fällen die einfache Dauerunterbindung am Orte der Wahl vorgenommen, wie auch in meinem Falle mit Erfolg und ohne Hirnerscheinungen, und es ist darum nicht gut $z u$ verstehen, warum $P$ erthes gerade seinen auch gün- 
stig verlaufenden Fall, in dem er mit seiner komplizierten Methode die Carotis verschloß, für diese als "Erfolg buchen will“.

Für unsertherapeutisches Vorgehen bei Operationen anden $\mathrm{C}$ a rotiden möchte ich aus dem Vorstehender folgende Richtlinien zusammenstellen:

I. Es ist, je nach dem erstrebten Zwecke unseres Vorgehens, stets das anzuwenden, welches am schonendsten diesen Z weck erreicht. Man sollte in jedem Einzelfalle also nicht von Carotidenunterbindung sprechen, sondern genau auch in der Literatur angeben, ob es sich um die Carotis communis, interna, externa oder mehrerer gleichzeitig handelt. Für das Gehirn am gefährlichsten ist dieAusschaltungderGefäße in folgender Abstufung: a) C. interna bzw. communis + externa, b) C. interna, c) C. communis, d) C. externa, diese nur in seltensten Fällen und dann doppelseitig mehr als einseitig.

2. Zur genauen Orientierung über die vorliegenden Verhältnisse tut man meistens gut, sich die Gabelung freizulegen und in zweifelhaften Fällen von ihr ausgehend zu sehen, welche Wirkung gegebenenfalls die vorübergehende Blutsperre auf die vorliegenden Symptome wie Blutung, Pulsation usw. haben mag.

3. Unter der Voraussetzung, daß jede Gefä $B$ operation a u d as $S c h$ onendstedie Gefä $B$ wandundihren Inhalt berücksichtigt und behandelt, daß jede unnütze Abklemmung, zumal mit starren Klemmen, jede unnütze Entblößung von Adventitia, die die Blutgefäße trägt, kann die te mporäre Sperre mit geeigneten Klemmen oder Umschnürungen als verhältnismäBig harmloser Eingriff für das Gehirn gelten, doch darf sie nicht unnötigerweise lange ausgeführt werden. Die temporäre Sperre ist stets statt der Dauerunterbindung zu wählen, wo man glaubt, mit ihr auch für die Zukunft auszukommen. In zweifelhaften Fällen, z. B. Nachblutungsgefahr, wähle man lieber die Dauersperre oder eine andere, alle Gefahren ausschließende Operation.

4. Das Ideale ist für das Gehirn natürlich die $\mathrm{G}$ ef ä $\mathrm{Bn}$ a h t seitlich oder zirkulär nach Resektion unter temporärer Sperre. Der Wert der Gefäßnaht besteht natürlich in erster Linie in der sofort nach der Operation völlig oder doch wenig. stens in zureichendem Maße wieder hergestellten Zirkulation für 
das Gehirn, sodann aber auch darin, daß sich nach ihr als im strömenden Blute liegend, weniger leicht wandständige Thromben bilden. Diese Strömungseinflüsse können selbst leichte Infektionen überwinden, doch bleibt es immer gewagt, in inf $\mathrm{i}$. zierter Wunde, gerade an den Carotiden Gefä $B$. nähte a uszuführen. Gefahren und Vorteile sind hier besonders schwierig gegeneinander abzuwägen; es genügt nicht allein ein tüchtiger Operateur zu sein, der die Technik beherrscht, sondern vorsichtiges Abwägen, besonders in Dringlichkeitsfällen muß das Handeln leiten. Eine nach einer Gefäßnaht einer C. interna oder communis sich bildende Thrombose kann als solche wie auch besonders leicht durch Embolie für das Gehirn deletär werden. Kompliziertere Operationenanden Gefä Ben wie die Anastomose $z$ wischen $\mathrm{C}$. externa und interna, wie sie $Q \mathrm{u}$ énu vorgeschlagen hat, oder Umlagerung der C. externa zur Ausgleichung einer Lücke zwischen $C$. communis und interna, wie sie $\mathrm{Ed}$. Rehn vorschlug, sind nur in ganz seltenen Fällen (s. Fall 7) erlaubt oder möglich, die Gefahren der Thrombose und Infektion scheinen mir bei solchen Verfahren besonders gro $B$ zu sein.

6. MuB eine Unterbindung einerder Carotiden vorgenommen werden, so sind nicht komplizierte Verfahren, wie Perthes vorschlug, unter Zurücklassung einesgrößeren Fremdkörperszu wählen, sondern wirkommen mit deneinfachen altbewähr. ten Unterbindungsverfahren nicht nur aus, son. dern sie sind allen anderen vorzuziehen. Nicht die Fadenunterbindungmit nachfolgender GefäB. wandschädigung ist das eine etwaige Thrombose auslösende Moment, sondern die Infektion, und es ist diese, die bei allen GefäBoperationen besonders zu vermeiden oder zuberücksichtigen is t! Die chirurgische Praxis, die durch eine Änderung des Unterbindungsmaterials eine günstigere Prognose zu schaffen suchte, lehrt bis jetzt auch nicht irgendeinen Vorteil (s. auch S moler, Zur Unterbindung der Carotis communis. Bruns' Beiträge 1912, Bd. 82).

7. Die Unterbindung der C. communis ist, wenn 
möglich, einige Zentimeter zentral der Gabelung vorzunehmen, die der $C$. interna und externa einige Zentimeter peripher der Gabelung, um bei allfallsigem Auswachsen eines Thrombus die so wichtige Gabelungsstelle nicht zu gefährden.

8. Ist zu befürchten, daß sich bereits ein Thrombus in dem zu unterbindenden oder sonstwie anzugehörenden Gefäße befindet, so ist nach Möglichkeit zu versuchen, vor Manipulation an der Läsionsstelle, besonders also bei kommunizierenden Hämatomen, den peripheren Gefäßabschnitt wenigstens temporär zu sichern, um das Einschwemmen in das Gehirn, das so wichtige Endorgan, zu verhüten, wie auch Perthes vorschlägt.

Andere $\mathrm{MaBnahmen}$, die Gefahr der Carotidenunterbindung zu verringern, können manchmal zweckmäßig angewandt werden, doch ist ihr Wert stets für den Einzelfall abzuschätzen. So wird die besonders von Jordan, Smoler und anderen empfohlene vorherige Drosselung der C. communis wohl nur als Vorbereitung vor Exstirpation maligner Geschwülste angebracht sein; bei kommunizierenden Hämatomen wird sie ihre Gefahren haben, da eine Freigabe des Blutstroms leicht zu embolischen Prozessen aus dem Blutsack führen kann. Für Blutungen, Verletzungen, intrakranielle Hämatome ergibt sich die Beschrän. kung von selbst, im Krieg meist schon durch den Mangel an Zeit und Beobachtungsmöglichkeit. Die Vena jugularis int. gleichzeitig mit der Carotis communis oder interna $a b z u b$ inden, um für das Gehirn die Blutzufuhrschwankung herabzusetzen, hat bisher praktisch sich noch nicht als nützlich oder ausschlaggebend erwiesen. Ihr $E$ influB $l$ ä $B \mathrm{t}$ sich a uch wohlschwerim Einzelfalle nachweisen. Nicht unbedenklich erscheint mir bei etwaiger bestehender Infektion die Gefahr, ein Opfer der Venenthrombose werden zu können.

$\mathrm{DaB} Z$ Zahlenangaben oder Einzelveröffent lichungen günstiger Fälle, zumal aus dem Kriege und zumal dann, wenn sie ohne nähere Angaben über Art der Verletzung, Operationsanzeige, Vorhandensein einer Infektion u. a. $\mathrm{m}$. gemacht werden, nur selten $z u$ verallgemeinernderen Schlüssen berechtigen, liegt auf der Hand. Man könnte 
da manchmal zu ganz unglaublichen Schlüssen kommen, z. B. wenn man $\mathrm{P}$ ir og of $\mathrm{f} s$ Angabe, daß er unter 14 eigenen Carotis communis-Unterbindungen 9 ,volle Erfolge" gehabt habe, vergleichen wollte mit der von Hotz ganz beiläufig ohne nähere Angaben hingeworfenen Bemerkung, der Ed. Rehn aber doch einen erheblichen Wert beimessen zu dürfen glaubt, vergleichen wollte, daß nämlich $\mathrm{Hotz}$ bei 6 Unterbindungen der C. communis, die er an Durchgangsverwundeten beobachtete, 5 mal Halbseitenlähmung gesehen habel Ich meine, weniger wäre in diesem Falle mehr gewesen, um Trugschlüssen vorzubeugen.

Bei den Kriegsoperationen müssen wir auch immer bedenken, daß wir doch meistens nur die verhältnismäßig günstigen Gefäßverletzungen zur Behandlung bekommen, daß eine ganze Anzahl von Carotisverletzungen, und zwar je später sie zur geeigneten Behandlung also vom Kampfplatz bis zur endgültigen Versorgungsstelle kommen, desto mehr den Folgen der Carotisverletzung erlegen sind, sei es durch Frisch- oder Nachblutungen, sei es durch Hirnstörungen infolge der Gefäßunterbrechung, ohne daß deren eigentliche Ursache erkannt würde.

Ich habe versucht, unter den obigen Gesichtspunkten die Literatur der beiden letzten Jahrzehnte auf Carotidenunterbindungen usw. durchzusehen, habe aber nach dem Studium dieser Mitteilung ersehen, daß es eine nicht lohnende Mühe war und stehe darum auch ab, die Fälle hier kasuistisch oder statistisch zu verwerten, denn $\mathrm{kaum}$ eine derKrankengeschichten gibt Auskunft über alle der oben gestreiften in Betrachtzuziehenden Punkte, auch die meinigen nicht. Dennoch muß ich die mir zurzeit zur Verfügung stehenden Eigenerfahrungen kurz anfügen, um einzelne meiner Sätze in der vorstehenden Abhandlung zu stützen.

Leider fehlen mir über die vielen Unterbindungen der Carotis communis, die ich in der Türkei als Voroperation oder notgedrungen bei den so mächtigen Karzinomen und Sarkomen der Halsgegend ausführte, Aufzeichnungen, doch glaube ich sicher zu sein, daß bei fast allen diesen, ältere Leute betreffenden Fällen, wenn sie zugrunde gingen, das sog. freie Intervall fehlte (s. Fall 9). 
Zwecks Behandlung der genuinen Epilepsie habe ich in drei Fällen beide Carotides communes, wie empfohlen, mittels dicken Zwirns soweit gedrosselt, daß noch eben der periphere Puls sich feststellen ließ, zwar ohne jeden therapeutischen Erfolg, aber auch ohne jede Schädigung am Gefäß oder Gehirn (der Jugendlichen!).

Aus dem Weltkriege stehen mir auch nur die Kranken. geschichten a us den Jahren I9I7 und I9I8 zur Verfügung, natürlich als Kriegskrankengeschichten auch nur unvollständig. Was mir also an Aufzeichnungen vorliegt, gebe ich kurz nachstehend wieder:

F a 11 I. Soldat Sch., 30 J., kräftiger Mann, Granatsteckschuß vor 6 Stunden mit markstückgroßem Einschuß auf der Mitte des rechten Kopfnickers, Schwellung und Schwirren bis zum Schlüsselbein. Splitter steckt nach dem Röntgenbild rechts vor dem 6. und 7. Halswirbel, ein anderer in der rechten Augenhöhle. Keine sonstigen Störungen.

Op. Freilegung der Carotis communis unterhalb der Schilddrüse. Temporäre Ligatur auf Jodoformmullstück genügt nicht, daher temporärer Verschluß mit Wiet ingscher $G$ efä $B \mathrm{klemme}$, dic die Vena jug. mitfaßt, um Luftembolie vorzubeugen. Vorwärtsarbeiten peripherwärts. Abbinden der Vena fascialis und später auch des zerrissenen Endes des Vena jug. int., in deren Lumen der Splitter steckt. Die Blutung ist schwer mit einem Daumen zu stillen, da die Car. com., auf $2 \mathrm{~cm}$ zerfetzt, aus beiden Enden blutet. Abbinden beider Enden mit Zwirn, wobei die Gabelung nicht zu Gesicht kommt. Die hinten bestehende, schmale Carotidenbrücke wird nicht durchtrennt, um die Stümpfe nicht zurückschnellen zu lassen. Schichtennaht mit Mullstreifenableitung. Zwei Stunden nach Op. wird festgestellt, daß keinerlei Hirnstörungen bestehen. Eine nach 5 Tagen auftretende beiderseitige Kieferhöhleneiterung stört den Wundverlauf nicht; nach 6 Wochen Abtransport.

Epikrise: Frische Granatsplitterzerreißung der rechten Carotis communis und V. jug. int. Abbindung aller $S t u ̈ m p f e$ mit $Z$ wirn, keinerlei Hirnstörungen. Weg zwischen Externa und Interna frei, glatte Wundheilung.

Fall 2. Soldat W., 28 J., mittelkräftiger Mann, Granatsplitter vor 24 Stunden am linken innern Augenwinkel eingedrungen, einer der Splitter sitzt nach Röntgenbild in der Schädelhöhle hinter der Augenhöhle, die Wunde heilt gut, doch bildet sich pulsi erender Exophthalmus mit Amaurose links ohne sonstige Gehirnstörungen.

Die Frage, ob Unterbindung an der Carotis vorgenommen oder 
verschoben werden soll, wird dadurch entschieden, daß etwa 6 Wochen nach der Verletzung plötzlich eine starke pulsierende Blutung aus Nase und Mund auftritt. Während ich gerade in Fall 7 die Car. com. zum zweitenmal unterbinde, wird diese Blutung gemeldet. So hat $W$. das Glück, daB ärztliche Hilfes sofort vorhanden ist. Während ich mit der linken Hand linksseitig am Halse abdrücke, wodurch die Blutung aufhört, lege ich mit der rechten Hand die Car. com. frei, sie wird etwa $2 \mathrm{~cm}$ herzwärts der Gabelung mit $Z$ wir $n$ abgebunden, in der Kontinuität. Da die Op. nicht einwandsfrei aseptisch vor sich gehen konnte, wird die Operationswunde mit Jodtinktur ausgewischt, und über Jodoformmullstreifen vernäht. Primäre Heilung, keinerlei Gehirnstörung, das Auge tritt zurück, pulsiert nicht mehr. Der kleine Granatsplitter ist mit der Blutung durch die Perforationsstelle zur Nase ausgestoßen. Pat. geheilt entlassen. Nach 6 Monaten berichtet er, daß beim Bücken noch Schwirren und leichter Schwindel auftrete. Objektiv war in der Tiefe noch Schwirren mit dem Hörrohr nachweisbar. Die Amaurose bleibt natürlich (Opticusatrophie).

Epikrise: Pulsierendes $\mathrm{H}$ ämatom der intrakraniellen Car. int. im Sinus cavernosus. Spontandurchbruch nach der Nasenhöhle, einfache Ligatur der Car. com. mit Zwirn, k e in e r lie Hirnstörung bei primärer Wundheilung.

F all 3. Soldat L., $25 \mathrm{~J}$, frischer GewehrdurchschuB mit kommunizierendem Hämatom der linken Car. com. und $V$. jug. int. o h ne H irnstörungen, hat sehr stark geblutet, das Hämatom reicht bis unter den Kieferrand.

Op. Um die Gefahr der Blutung nach außen herabzumindern und andererseits einen größeren Blutverlust bei der in Aussicht genommenen größeren Gefäßoperation vorzubeugen, wird die Car. com. und $V$. jug. int. dicht am Hämatom zentral mit Zwirn unterbunden durch Sonderschnitt, um die Ausräumung später vorzunehmen. $\mathrm{N}$ a $\mathrm{ch}$ der Ligatur gutes Befinden, ohn e Hirnstörung. Die Schnittwunde heilt primär. Militärische Rücksichten gebieten den Abtransport vor der 2. Operation.

Epikrise: Provisorische Unterbindung, der Car. com. und Vena jugularis bei pulsierendem Hämatom ohne primäre oder sekundäre Hirnstörung bei aseptischem Verlauf der Voroperation.

Fall 4. Soldat M., $28 \mathrm{~J}$,, schwere $Z$ ertrümmerung des Unterkiefers mit weiter Zerreißung der Weichteile. Tiefe jauchige Wunde, in der oberen Halsgegend mit viel Knochengrus und wiederholten Nachblutungen, die zu schwerer Anämie innerhalb 4 Tagen geführt hatte. Da mir die Unterbindung der Car. ext., wie ich sie sonst prophylaktisch bei schweren Kieferschüssen auszuführen pflegte, in diesem Falle nicht ausreichend erscheint, unterbinde ich 
von neuem Schnitt aus, etwa $2 \mathrm{~cm}$ zentral der Gabelung, die Car. com. Gehirnerscheinungen treten danach nicht auf, die Blutungen stehen, die Schnittwunde heilt glatt, die Schußwunde reinigt sich, der Pat. wird nach 2 Monaten zur Kieferplastik abtransportiert.

Epikrise: Prophylaktische Unterbindung der Car. com. mit $Z$ wirn ohne nachfolgende Hirnerscheinung.

F all 5. Soldat M. kommt ro Stunden nach der Granatsplitterverletzung in das Lazarett in bewuBtlosem Zustand, aus dem er sich indessen nach einigen Stunden erholt (Gehirnerschütterung). Der Warzenfortsatz ist zertrümmert, aus dem rechten Gehörgang fließt Blut mit Knochentrümmern, doch anscheinend kein Gehirn, das rechte Auge blutunterlaufen. Das Röntgenbild zeigt eine Knochenzertrümmerung des rechten Felsenbeins, ein bohnengroßer Geschoßsplitter sitzt an der Schädelbasis nahe der Mittellinie rechts. Unter antiseptischem Verband mit Glyzerinjodoform gute Erholung ohne Fieber, bis am 12. Tage plötzlich aus dem eitrig sezernierenden Ohre eine starke Blutung erfolgte.

Diese steht auf feste $\mathrm{T}$ amponade, doch wiederholt sie sich bei Lockerung der Stopfung. Sie stammt nach Menge und Aussehen, offenbar aus der Car. int. an der Schädelbasis, und zwar aus ihrem Verlauf im Felsenbein. Ein neuerliches Röntgenbild zeigt $\mathrm{ke}$ inen GschoBsplitter mehr, dieser ist offenbar bei einer der Spätblutungen herausgeschwemmt und unbemerkt fortgeworfen. Der Blutverlust hat den an und für sich kräftigen Menschen erheblich geschwächt. Es wird nun die $\mathrm{Ca}$ rot is in terna $\mathrm{rech}$ t $\mathrm{s} f \mathrm{e}$ igeleg t a $\mathrm{m} \mathrm{Halse}$ und mit Zwirn unterbunden, das Loch im Felsenbein erneut ausgestopft. Vom Augen blick der Operation an ist der Kranke bewuBtlos bei gutem Puls, erholt sich aber nach zwei Tagen und beginnt zu trinken und Abwehrbewegungen zu machen, es besteht motorischeAphasie(l) und völlige $\mathrm{H}$ albseitenlähmung. Trotz der Maßnahmen wiederholt sich die Blutung nach abermals 8 Tagen und dies noch weitere dreimal, trotzdem die feste Jodoformstopfung mittels enger Zwirnnaht der Haut festgehalten wird. SchlieBlich gelingt es doch, auf diese Weise der Gefahr Herr zu werden. Innerhalb von $2 \mathrm{M}$ on ate $\mathrm{n}$ geht die Halbseitenlähmung einschlieBlich der Sprach. störung, und zwar manchmal in ruckweiser Besser u n g zurück. Die Wunde schließt sich völlig nach Abstoßung einiger Knochensplitter, so da $B$ der Kranke 8 Wochen nach der letzten Blutung in bestem Zustand mit mäßiger Halbseitenparese in die Heimat entlassen werden kann.

Epikrise: Unterbindung der an der Basis cranii rechts verletzten $\mathrm{Carotis}$ interna $\mathrm{nach} 12 \mathrm{Tagen}$ bei einem stark ausgebluteten Mann. Erfolg bezüglich der Blutstillung unzureichend, 
da die Versorgung aus der anderen Carotis bereits hergestellt, eine Unterbindung auch dieser aber das Leben gefährdet hätte. Dennoch genügte die Unterbindung an derselben Seite bei dem a u s g e b l u te-


chende Hirnstörung hervorzurufen. Gefäßnaht natürlich bei Lage der Verletzung ausgeschlossen.

Eine Embolie nach Thrombenbildung ist wohl $n i c h t$ a nzunehmen dem ganzen Entstehen des Krankheitsbilds nach. Schußwunde infiziert.

F a 11 6. Soldat L., $22 \mathrm{~J}$., kommt 12 Stunden nach schwerer Blutung aus Granatsplitterschuß, der den rechten Warzenfortsatz zertrümmert hat, in das Lazarett. Die zerrissene Wunde führt gegen die Schädelbasis durch starke nicht pulsierende Schwellung der $\mathrm{r}$. Halsseite. Dem Röntgenbild nach muß3 die Car. int. nahe der Basis, wo der Splitter sitzt, lädiert sein. Es besteht ke in e primäre $L a ̈ h m u n g$ oder BewuBt seinsstörung.

Op. Da dauernd in den Verband Blut aus dem Einschuß sickert, trotz fester Übernähung über Tamponade, wird nach 4 Stunden in leichter Narkose dic Car. com. freigelegt und diese, um auch eine Externablutung möglichst auszuschalten, einfach mit Zwirn unterbunden. Nach der Op. wird sofort links $\mathrm{H}$ albseitenlähmung mit Somnolenz beobachtet, die nach 12 Stunden über Coma zum Tode führt. Leichenbefund fehlt.

Epikrise: Schwere Anämie, durch Blutung aus verletzter Car. int. Unterbindung der Communis in der Kon. tinuität durch $\mathrm{Zwirn}$, ohne Intervall Halbseitenlähmung und Tod. Schußwunde infiziert. (Vielleicht hätte eine Bluttransfusion die schweren Folgen vermeiden können, doch war die Zeit zu dringlich bemessen.) Embolie oder Thrombose als Ursache ist wohl nicht anzunchmen. Die allgemeine Anämie vermehrte die lokale des Gehirns, so da $B$ absolut die Blutzufuhrungenügend werden muBte.

F all 7. Soldat G., 20 J., Granatsplittersteckschuß in der linken Halsseite, kommt nach ro Stunden mit übernähter Tamponade vor dem linken Kopfnicker. Außer H or n e r schem Sympathikuskomplex links keinerlei nervöse Störung. Nach Lösung der Naht e rne ute Blut ung. Hohes Fieber. Freilegung der Car. com. und Vena jug. in infizierter Wunde. Beide zerrissenen Gefäße (Vagus?) werden in Höhe der Schilddrüsen doppelt unterbunden mit Zwirn, der Splitter entfernt. Die Blutung steht ohne jede Hirnstörung. Wohlbefinden, doch dauernd Eiterung und Fieber, trotz Kopfruhigstellung erfolgen nun in Abständen von jedesmal 10 bis 12 Tagen arterielle Spätblutungen aus der Wunde, die jedesmal erneute Freilegung und erneute Unterbindung der Communis und zwar ihres peripheren Teiles nötig machen, während der zentrale Teil in Ord- 
nung war. Die Blutungen erfolgten stets unter AbstoBung des Seidenfadens durch arteriitische Wandnekrose, bei welcher ja, wie auch Dietrich nachwies, eine eigentliche Thrombenbildung (Verschlußpropf) sehr gering bleiben kann. Nach der letzten Unterbindung, die in das Gebiet der Gabelung fallen mußte, traten septische Erschei$\mathrm{n}$ ung e n mit Verschlechterung des Blutbefundes sehr in den Vordergrund. Im Laufe der nächsten Tage bildete sich linksseitige Amaurose und Panophthalmie und rechtsseitige Halbseitenlähmung. Dann trat der Tod unter schweren Lungenerscheinungen ein.

Epikrise: Konfluierende Bronchopneumonie beiderseits. Die linke Carotis interna ist von der Unterbindungsstelle bis zum Eintritt in den Sinus cavernosus mit flüssigem Eiter gefüllt, ohne eigentliche Thrombose. Es lag also eine Endarteriitis purule nt a vor, von der die Augenarterie sich eitrig gefüllt hatte. Weiterhin war die Carotis cerebralis frei und ohne Erweichungsherd. Also Carotidenunterbindung anfangs ohne Schaden, bis die aufsteigende Endarteriitis die Verbindung $z$ wischen Carotis ext. und int. durch Verlegung der A. ophthal$m$ ic a a f hob und so unter zunehmender Anämie die Halsseitenstörung geschaffen wurde. Hätte man den ungünstigen Verlauf voraussehen können, wäre vielleicht durch eine rechtzeitige Anastomose zwischen Car. ext. und int. bei gleichzeitigem Offenlassen des eiternden Lumens Rettung zu bringen gewesen.

F a 11 8. Soldat W., $20 \mathrm{~J}$., durch Handgranate dreieckiger Einschuß hinter dem linken Kopfnicker, Splitter steckt nach Röntgenbild links neben der Wirbelsäule in Höhe des Unterkieferwinkels. Schwirrendes pulsierendes Hämatom, im oberen Halsdreieck mit Schluckbeschwerden und Vorbuchtung nach der linken Rachenwand. Am folgenden Tage Temperatursteigerung.

Op. I4 Stunden nach der Verletzung, Freilegung der Gabel ung der Car. com., deren Scheide durch Blutung durchsetzt ist. Starke Blutung, die bei weiterem Vorgehen eintritt, wird durch Fingerdruck gestillt. Die in nere Jugularvene ist quer zerris sen und wird doppelt mit $Z$ wirn unterbunden. In dem Blutsack zeigt sich die Carotis externa ebenfalls völlig durchtrennt, doch ist das zentrale Ende durch kolbenförmigen Thrombus geschlossen und ragt stark pulsierend frei in den Blutsack. Dieses, wie auch das periphere Ende, das ebenfalls nicht blutet, werden einzeln mit $Z$ wirn unterbunden. Eine arterielle Blutung kommt nur a u s der Ca rot is interna, die bis auf ein kleines Band zerrissen ist. Beide Enden sind ziemlich eng kontrahiert und bluten beide, besonders wenn man den Druck auf die Communis nachläßt. Da die Naht in der großen Höhle, in der die Gefäße ohne Halt zu haben, freiliegen würde, und da zudem Infektion besteht, werden beide Interna-Stümpfe mit Zwirn unterbunden. Der Splitter wird unter der Rachenschleimhaut von der Wunde her entfernt. 
Sofort nach der Narkose wird Halbseitenläh$\mathrm{mung}$ rechts festgestellt mit schnarchendem Atem, ohne völlige Bewußtlosigkeit, aber S p r e chstör ung. Er reagiert auf Anruf durch Augenöffnen. Die linke Pupille ist maximal erweitert, die rechte minimal verkleinert. Am nächsten Tage Coma, am folgenden Morgen tot.

Epikrise: Bei der Sektion embolische Erweichung der linksseitigen Stammganglien, Thrombus in der Carotis interna, dieser hatte sich offenbar im Lumen an der (infizierten) Läsionsstelle gebildet und wurde durch das Hantieren am Gefäß zum Embolus und zur Todesursache. Die Lage war ungünstig: Ligatur der Car. ext. und int., die infiziert und thrombosiert ist, -.- ein lntervall und doch Embolie.

I a 11 9. (Frühere Friedensbeobachtung.) 48 Jahre alter nicht ausgebluteter, aber arteriosklerotischer Mann, mit mächtigem Karzinom der r. Augenhöhle mit Einwucherung auf das Stirnhirn, es wird vorbeugend die Car. com. durch Sonderschnitt unterbunden, danach das Karzinom ausgeräumt, wobei doppelte Umstechung des Sinus long. nötig war. N a ch der Op. bleibt $\mathrm{Pa}$ tient komatös ohnefreies Intervall, rechts gelähmt, links agiticrt. Tod nach 2 Tagen. Sektion; Keine Thrombose oder Embolie in den $G$ ef äB en. Makroskopisch kein deutlicher Unterschied in den beiden Großhirnhälften.

F a 11 10. Knabe Mustapha, I2 J. Dieser Fall wurde bereits früher mitgeteilt durch meinen Assistenten Dr. Emin (siehe Münchner med. Wochenschr. 1907). Es tritt hier nach Unterbindung der Car. com. und Vena jug. int. bei infiziertem Hämatom durch Stichverletzung innerhalb i2 $\mathrm{Stunden}$ rechtsseitige Halsseitenlähmung auf. Die Feststellung eines freien Intervalls ist $\mathrm{nicht}$ gesichert, da der kleine Patient nach der Narkose und starkem Blutverlust weiter schläft und erst etwa I2 Stunden nach der Unterbindung klinisch die Hirnerscheinungen mit Coma beobachtet werden. Nach schwerem Krankheitsbild ist nach 8 Monaten jede Gehirnerscheinung verschwunden. 